Purdue University

Purdue e-Pubs

$1-1-2002$

\title{
Concentration Fields in the Solidification Processing of Metal Matrix Composites
}

B Moussa

JE. Simpson

S V. Garimella

Purdue University, sureshg@purdue.edu

Follow this and additional works at: http://docs.lib.purdue.edu/coolingpubs

Moussa, B; Simpson, J E.; and Garimella, S V., "Concentration Fields in the Solidification Processing of Metal Matrix Composites" (2002). CTRC Research Publications. Paper 80.

http://docs.lib.purdue.edu/coolingpubs/80

This document has been made available through Purdue e-Pubs, a service of the Purdue University Libraries. Please contact epubs@purdue.edu for additional information. 


\title{
Concentration Fields in the Solidification Processing of Metal Matrix Composites"
}

\author{
Bechir Moussa \\ Department of Mechanical Engineering \\ University of Wisconsin - Milwaukee \\ Milwaukee, WI 53201 USA \\ James E. Simpson ${ }^{\dagger}$ and Suresh V. Garimella \\ School of Mechanical Engineering \\ Purdue University \\ West Lafayette, IN 47907-1288 USA \\ Ph: (765) 494-5621 Fax: (765) 494-0539
}

\begin{abstract}
A numerical investigation of the solidification of a binary alloy (Al-1.0 wt.\% $\mathrm{Cu}$ ) around cylindrical fibers with different fiber layouts and thermophysical properties was undertaken to gain insight into the processing of fiber-reinforced metal matrix composites (MMCs). The focus of this study was on solute transport and redistribution during the solidification process, and the resulting concentration fields in the solidified alloy matrix. Change of phase in the alloy was formulated using a modified version of the temperature-transforming method for the energy equation. A source term that accounts for the solute rejection at the interface was incorporated into the solute concentration equation to model solute redistribution at the interface. Detailed results were obtained from the numerical simulations of low- (alumina) and high- (copper) conductivity fibers in inline and staggered configurations. Effects of the fiber pitch (longitudinal spacing) and transverse spacing were investigated. Higher concentrations of solute were seen to accumulate around copper fibers than for alumina fibers. With an initial, uniform concentration of $1.0 \mathrm{wt} . \% \mathrm{Cu}$ in the melt, the maximum-recorded solute concentration in the domain for alumina fibers was $1.26 \%$ while that for copper fibers was $3.11 \%$. For inline fibers, increasing the fiber pitch beyond a critical value did not change the overall shape of the local solute distribution around the fibers: the critical pitch for alumina fibers was found to be roughly 2.5 fiber diameters while that for copper was 2 fiber diameters.
\end{abstract}

\footnotetext{
${ }^{\pi}$ Submitted for possible publication in International Journal of Heat and Mass Transfer, November 2001, and in revised form, April 2002.

${ }^{\dagger}$ Postdoctoral Research Associate

* Associate Professor, to whom correspondence should be addressed, E-mail: sureshg @ ecn.purdue.edu
} 


\section{NOMENCLATURE}

A coefficient in the finite volume equation

$b \quad$ wall temperature cooling rate

$c_{p} \quad$ specific heat capacity at constant pressure

C species concentration

$D_{l} \quad$ species diffusion coefficient (liquid $\mathrm{Cu}$ in liquid $\mathrm{Al}$ )

$D \quad$ fiber diameter

$f \quad$ volume fraction

$H \quad$ transverse spacing

$h_{e} \quad$ reference specific enthalpy

$k \quad$ thermal conductivity

$L \quad$ length of simulation domain

$S \quad$ fiber pitch

$t$ time

$T \quad$ temperature

$v \quad$ front speed

\section{Greek Symbols}

$\alpha \quad$ thermal diffusivity

$\delta \quad$ diffusion boundary layer thickness

$\kappa_{p} \quad$ partition coefficient

$\eta \quad$ ratio $\left(=k_{f} / k\right)$ used in Eq. (3)

$\rho \quad$ density

$\Delta H \quad$ enthalpy of freezing

\section{Subscripts}

c cold

f fiber

i initial condition

1 liquid

m melting point 
S solid

\section{Superscripts}

e effective

n time step $n$

\section{INTRODUCTION}

Composite materials are engineered combinations of two or more materials in which tailored properties are achieved by systematic combinations of the different constituents. Various types of engineered composites are prevalent in the industry, including polymer matrix, ceramic matrix and metal matrix composites. Metal matrix composites (MMCs) are made of a continuous metallic matrix and one or more discontinuous reinforcing phases. The reinforcing phase may be in the form of fibers, whiskers or particles. For the case of fiber reinforced MMCs, typical fiber diameters (e.g. alumina, silicon carbide) range from 5 to $25 \mu \mathrm{m}$ [1]. The material, amount and size of the reinforcing phase are usually dependent on the desired property for a specific application.

Modern MMCs offer the advantage of being engineered to conform to a particular set of specifications on weight, stiffness and wear resistance. In addition, spatial variation in properties may be achieved by modifying the location and composition of the reinforcing material. Compared to monolithic metals, MMCs have: higher strength-to-density and stiffness-to-density ratios, superior fatigue and creep properties, better abrasion resistance and lower coefficients of thermal expansion. Advantages of MMCs over polymer-matrix composites and ceramic-matrix composites include higher temperature capability and higher electrical and thermal conductivities.

Metal-matrix composites are used in spacecraft, commercial airliners, electronic substrates, automobiles, sports equipment, high-temperature heat exchangers and other 
mechanical applications such as pistons, cylinder liners and bearings [2]. Different values for the stiffness, strength, density, and thermal and electrical properties can be obtained by appropriate choice of the matrix alloy, reinforcement material, volume and shape of the reinforcement, location of the reinforcement, and fabrication method of these composites. Regardless of the specific components, aluminum composites offer the advantage of low cost over most other MMCs. In addition, they offer excellent abrasion resistance, superior high-temperature performance and the ability to be formed and treated through conventional means.

A variety of methods for producing MMCs have been developed, including gravity or pressure die-casting, centrifugal casting, stir-casting and pressure infiltration. Such methods may be considered liquid-state processes, as the metal matrix is in a molten state at the beginning of the production cycle. Solidification in such liquid-state methods is a complex transformation and the details of the process need to be understood in order to successfully produce MMCs. Factors such as propagation and stability of the solidifying front in the presence of the reinforcement, movement of the discontinuous phase (fiber/particle pushing and settling), thermo-chemical reactions at the solidification front and interfacial bonding between the metallic matrix and reinforcement, all affect the properties of the final product.

Extensive numerical and experimental studies have been reported for the solidification of either pure metals or binary alloys. Early models ranged from the simple one-dimensional analytical solution involving only heat conduction (as described in [3]) to the more recent development of solidification models that account for the simultaneous heat, mass and momentum transport as well as the mushy zone, shrinkage, and other phenomena. Comprehensive reviews on the subject are available (for example, $[4,5]$ ). Fewer studies have been reported which are targeted specifically at detailed simulations of MMC processing. One of these processing techniques uses a dispersion of ceramic or metallic particles in metallic 
melts. In this method, the reinforcing particles are stirred into the alloy melt, and the homogeneous mixture obtained is allowed to solidify [1]. The final microstructure of the solidified MMC depends greatly on the stirring speed [6]. Another more common and economical method for the synthesis of MMCs is the preform-based cast processing. In this process, fiber preforms are infiltrated under low pressure with the alloy matrix and allowed to solidify [7]. A number of metallurgical studies from the viewpoint of understanding microstructures of MMCs have been reported [1, 8, 9]. Several researchers $[7,10,11]$ presented calculations and limiting-case expressions for the infiltration kinetics and temperature distribution during infiltration of fiber preforms. Mortensen et al. [10] obtained a similarity solution for the one-dimensional heat conduction equation. The domain of interest was divided into subregions containing as-infiltrated fibers, fibers with a liquid metal matrix, and fibers with a solidifying matrix. The rate of infiltration was constant and determined from D'Arcy's law. Appropriate matching conditions were posed at each boundary, and closed-form solutions were obtained for simple cases. The time scale of the analysis was such that "instantaneous" heat transfer to the fibers from the melt was assumed. In the last phase of this work, the analysis was generalized to include multidimensional heat transfer due to non-adiabatic side walls. The analytical expressions developed in this work were validated by experiments performed by Masur et al. [11]. The experimental results exhibited very good agreement with the onedimensional analysis, but less satisfying agreement for the multidimensional case due to a lack of knowledge of the applied thermal boundary conditions. Similarity solutions were also obtained for infiltration with a binary eutectic matrix [7].

Such macroscopic analyses are useful for elucidating the bulk behavior during the synthesis of composite materials, but do not consider the finer-scale effects of the influence of the fibers on the thermal field and the shape of the propagating solidification front. Khan and 
Rohatgi $[12,13]$ numerically investigated the propagation of the solidification front around fibers as well as the thermal fields in the metal and fiber. A three domain approach was used one each for the fiber, liquid and solid phases. A "time" method was used to track the location of the solidification front. A coordinate transformation was employed to cope with the introduction of a cylindrical fiber domain into the cartesian domain representing the mold. Their results showed that the fibers have a strong impact on the shape and progression of the propagating solidification front. Fan et al. [14] performed similar computations. These studies considered heat transport by conduction only; the effects of convection in the melt were neglected and mass diffusion did not have to be addressed for the pure metals studied. The disadvantage of the solution scheme employed in these studies is that, while being accurate, it is computationally expensive and difficult to extend to account for the effects of convection in the melt and the presence of solutal gradients.

More recently, MMC solidification has been investigated via numerical investigations of solidification of mainly pure metals within a domain with a fibrous phase. A pure aluminum matrix with a single fiber [15], a pure aluminum matrix and an aluminum alloy matrix with a fixed set of in-line fibers [16], and a pure aluminum matrix with both in-line and staggered fibers in a wide variety of distributions [17] were considered. The computational approach used was a modified enthalpy method, which accounts for both phase-change and the discontinuous fibrous phase. Results were obtained for single and multiple fibers and the solutions presented were obtained in significantly shorter computational times than comparable methods [12,13]. Effects of fiber spacing in inline and staggered configurations were obtained by Guslick et al. [17] and a critical fiber spacing beyond which the solidifying front was no longer affected by further increases in spacing was reported. The emphasis in these studies was on the effects of the fiber 
properties and configuration density within a domain on the thermal field and the solidifying front. Convection effects were neglected and solute concentration effects were not considered.

The aim of the present work is to advance these previous studies by considering the solidification an aluminum alloy matrix in the presence of a fiber phase. The fiber phase includes low- (alumina) and high- (copper) conductivity fibers in both inline and staggered configurations. A wide variety of fiber spacings are examined. The emphasis of this analysis is on the study of the solute concentration distribution, temperature profiles and influence of fiber configuration on solute concentration fields; for this study, the effects of shrinkage, fiber wetting and dendritic growth are neglected. The reinforcing fibers are stationary and solidification is normal to the axis of the fibers. The solid/liquid interface is assumed to be distinct. The effect of the fibers on the solute distribution in the solidified material is demonstrated.

\section{MATHEMATICAL FORMULATION AND NUMERICAL SCHEME}

The problem under consideration is the solidification of a dilute binary alloy (the matrix) in the presence of reinforcing fibers. These fibers have different thermal and physical properties from the matrix but do not undergo phase change, since their melting temperature is much higher than that of the matrix. This situation is encountered during the synthesis of metal-matrix composites by liquid-state processes such as pressure casting. A schematic diagram of such a process is shown in Fig. 1(a). Fig. 1(b) shows the problem domain, which is a closed twodimensional cavity with adiabatic top and bottom walls and a chilled wall at the left end. A solidifying aluminum-copper alloy is contained within the cavity, along with solid fibers interspersed throughout, at initial temperature $T_{i}$. Additional planes of symmetry are inherent in the problem and allow the domain to be reduced further to the smallest possible unit which is represented in Fig. 1(c). The dominant mechanism of energy and mass transport in the domain is 
diffusion; since the fiber volume fractions in this work are significant, and the characteristic scale of the domain is microscopic, convective velocities are negligible.

The governing equation for the conservation of energy, including the presence of the fibers and phase-change in the matrix is:

$$
\rho^{e} c_{p}^{e} \frac{\partial T}{\partial t}=\nabla \cdot\left(k^{e} \nabla T\right)
$$

Modeling of phase-change in the matrix material follows the temperature-transforming model of Zeng and Faghri [18]. Some slight modifications are needed to account for presence of the fibers (which do not change phase). The expression for effective heat capacity then becomes:

$$
\rho^{e} c_{p}^{e}=f_{f} \rho_{f} c_{p_{f}}+f_{l} \rho_{l} c_{p, l}+f_{s} \rho c_{p, s}+\rho_{l} \Delta H \frac{\partial f_{l}}{\partial T}+\left(\rho_{f} h_{f}+\left(\rho_{l}-\rho_{f}\right) h_{e}\right) \frac{\partial f_{f}}{\partial T}
$$

in which $f_{f}, f_{l}$ and $f_{s}$ are the volume fractions of the fiber, liquid alloy and solid alloy, respectively. A model for thermal conductivity is also needed. A general model for thermal conductivity applicable to mixtures in which the geometry is not known [3] is used for this study:

$$
k^{e}=k \frac{1+f_{f}^{2 / 3}(\eta-1)}{1+\left(f_{f}^{2 / 3}-f_{f}\right)(\eta-1)}
$$

The governing equation for the transport of solute throughout the liquid is given by:

$$
\frac{\partial C_{l}}{\partial t}=D_{l} \nabla^{2} C_{l}+S\left(T, C_{l}\right)
$$

The rejection of solute into the liquid at the solid/liquid interface as solid is formed is modeled via the source term appearing on the RHS of Eq. (4). Following the work of Swaminathan and Voller [19] and Voller et al. [20], who developed such a model for solute rejection by considering solidification in a microscopic control volume with zero back diffusion in the solid, this source term becomes:

$$
S\left(T, C_{l}\right)=\kappa_{p} C_{l} \frac{\partial f_{s}}{\partial t}+\frac{\partial\left(f_{l} C_{l}\right)}{\partial t}
$$


For the present study, isothermal phase change is assumed for the Al-Cu alloy due to the very low initial concentration of solute. Therefore, the melting temperature is considered to be constant and equal to the melting temperature of pure Al. The diffusion of solute in the solidified matrix material is assumed to be negligible; the concentration at which solid first solidifies is unchanged for all time. This assumption is reasonable due to the relatively low mass diffusivity of solid copper in solid aluminum when compared to the corresponding value for the liquid state $\left(D_{s}=1.4 \times 10^{-13} \mathrm{~m}^{2} / \mathrm{s} \ll D_{l}=7.2 \times 10^{-10} \mathrm{~m}^{2} / \mathrm{s}\right)$.

The initial and boundary conditions for the thermal field are shown in Figs. 1(b) and 2(a). Extraction of heat from the ingot is modeled by slowly decreasing the temperature boundary condition applied at the left-hand wall:

$$
T_{c}(t)=T_{c}^{0}-b \cdot t
$$

where, $T_{c}^{0}=670^{\circ} \mathrm{C}, b=256^{\circ} \mathrm{C} / \mathrm{s}$ is the cooling rate, and $t$ is in seconds. For the solute field, the initial and boundary conditions are such that the whole domain initially has a solute concentration $C_{i}$ uniformly distributed throughout the domain and no mass flux is allowed out of the cavity walls or through the fibers.

Discretization of the domain is by finite volumes in space with the Euler implicit scheme used for time. The final finite volume equation can then be written as:

$$
A_{P} T_{P}^{n+1}+A_{E} T_{E}^{n+1}+A_{W} T_{W}^{n+1}+A_{N} T_{N}^{n+1}+A_{S} T_{S}^{n+1}=a_{P} T_{P}^{n}
$$

in which:

$$
\begin{aligned}
& A_{E}=\frac{\Delta t}{(\Delta x)^{2}} k_{e}^{e}, A_{W}=\frac{\Delta t}{(\Delta x)^{2}} k_{w}^{e}, A_{N}=\frac{\Delta t}{(\Delta x)^{2}} k_{n}^{e}, \text { and } A_{S}=\frac{\Delta t}{(\Delta x)^{2}} k_{s}^{e} \\
& a_{P}=\left(f_{f} \rho_{f} c_{p_{f}}+\left(1-f_{f}\right) \rho_{l} c_{p_{l}}+\left.\rho_{l} \Delta H \frac{\partial f_{l}}{\partial T}\right|_{P}\right) \\
& A_{P}=a_{P}+A_{E}+A_{W}+A_{N}+A_{S}
\end{aligned}
$$


The relationship between cell liquid fraction and temperature needs to be specified so that the finite volume equations (7) can be solved. In isothermal phase change, there is a point discontinuity in liquid fraction: for material below the melting temperature $T_{m}$, the cell liquid fraction is zero, and for temperatures larger than $T_{m}$, the liquid fraction is unity. This point discontinuity cannot be handled numerically. Instead, the discontinuity is spread out over a small temperature range of $2 \varepsilon$. Incorporating this concept and accounting for the presence of the fibers yields the supporting relationship for liquid fraction as a function of temperature:

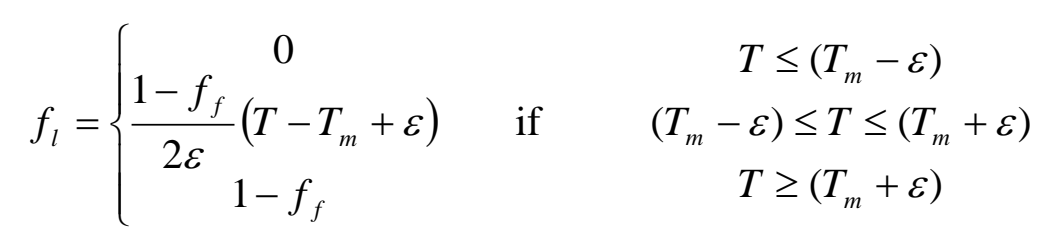

The temperature increment $2 \varepsilon$ is chosen small enough to assure that no more than two finite volumes undergo phase change at any given time. Values lower than this result in oscillations in the solution or a lowering of the latent heat released at the interface, while higher values result in the latent heat being released at a range of locations away from the interface. The discretized energy equation (7) was solved using Gauss-Seidel iteration with successive over-relaxation.

For the concentration equation, the finite-volume equation may be written as:

$$
A_{P} C_{P}{ }^{n+1}+A_{E} C_{E}{ }^{n+1}+A_{W} C_{W}{ }^{n+1}+A_{N} C_{N}{ }^{n+1}+A_{S} C_{S}{ }^{n+1}=f_{l}^{n+1} C_{P}{ }^{n}
$$

in which

$$
\begin{aligned}
& A_{E}=\frac{\Delta t}{(\Delta x)^{2}} D_{e}, A_{W}=\frac{\Delta t}{(\Delta x)^{2}} D_{w}, A_{N}=\frac{\Delta t}{(\Delta x)^{2}} D_{n}, A_{S}=\frac{\Delta t}{(\Delta x)^{2}} D_{s} \\
& b_{P}=\left(\kappa_{p}-1\right) f_{l}^{n}+\left(2-\kappa_{p}\right) f_{l}^{n+1} \\
& A_{P}=b_{P}+A_{E}+A_{W}+A_{N}+A_{S}
\end{aligned}
$$

Equation (10) is solved using Gauss-Seidel iteration with successive over-relaxation in a similar manner to the discrete energy equation (7). 
The solute concentration in the solid portion of a node does not change since solute transport in the solidified material is considered to be negligible. The following discrete integral is used to recover the solid concentration values in the cells as phase change occurs:

$$
C_{s}^{n+1}=\frac{C_{s}^{n} f_{s}^{n}+\kappa_{p} C_{l}^{n}\left(f_{l}^{n+1}-f_{l}^{n}\right)}{f_{s}^{n+1}}
$$

The program for the computations was written in ANSI standard FORTRAN 77 and the simulations were performed on a Compaq XP1000 alpha workstation (667 MHz CPU, SPECfp95 65.5). Computation times varied from $7 \mathrm{~min}$ to $185 \mathrm{~min}$ depending mainly on the computational domain, fiber configuration and spacing, and to a lesser extent, on fiber properties.

The grid-independence of the results obtained was verified by simulating the case of solidification in a domain with no fibers using a 300 x 30 grid and a finer, 600 x 60 grid, both with a time step of $\Delta t=7.815 \times 10^{-5} \mathrm{~s}$. Comparing profiles of solute concentration and temperature at, for example, $y=7.5 \mu \mathrm{m}$ and $\mathrm{x}=30 \mu \mathrm{m}$ revealed that the maximum difference between the two grids was identically zero for temperature and less than $0.87 \%$ for final solute concentration. The finer mesh predicted the interface to occur sooner (at smaller $x$ ) by one mesh point. This is not surprising since the front location is determined to within the numerically necessary temperature increment $2 \varepsilon$ in the liquid fraction vs. temperature relationship. The results obtained for solidification with no fibers was also used to validate the numerical predictions; the fronts were vertical and one-dimensional, typical of directional solidification in the absence of melt convection. The small temporal step size $\left(\Delta t=7.815 \times 10^{-5} \mathrm{~s}\right)$ was necessitated by the small scale of the domain and the low mass diffusivity in the melt (compared to the thermal diffusivity) to achieve adequate resolution of the concentration field. The reference time with which the above time step is nondimensionalized is $t^{*}=D^{2} / \alpha=3.096 \times 10^{-6}$ 
s where $D$ is the fiber diameter $(10 \mu \mathrm{m})$ and $\alpha$ is the thermal diffusivity $\left(3.23 \times 10^{-5} \mathrm{~m}^{2} / \mathrm{s}\right)$. Solidification times for all the simulations ranged from $0.4 \mathrm{~s}$ for three inline copper fibers to $6 \mathrm{~s}$ for six staggered alumina fibers.

\section{RESULTS AND DISCUSSION}

\section{In-line Fibers}

The effect of varying the fiber pitch $(S)$ on the final distribution of solute concentrations in the solidified alloy matrix for the inline fiber configuration is first examined. The computational domain for the simulations reported in this section is shown in Fig. 1(c). The fiber diameter used was $D=10 \mu \mathrm{m}$. The domain height was set at $15 \mu \mathrm{m}(H=1.5 D)$, and the domain length was fixed at $150 \mu \mathrm{m}(L=15 D)$ while the fiber pitch $S$ is allowed to vary $(S=1$, $1.5,2,2.5,3 D)$, corresponding to fiber volume fractions in the populated part of the domain of $26.2 \%, 17.4 \%, 13.1 \%, 10.5 \%$ and $8.7 \%$, respectively.

Figure 3 shows the concentration contours at five different fiber spacings with alumina fibers. The contours in the figures represent lines of constant solute concentration within the domain. Figure 3(a) illustrates the solute concentration contours in the solidified domain with three touching inline alumina fibers $(S=D)$ after a time of $0.3907 \mathrm{~s}$ has elapsed. At this time, the solidification front has already passed the part of the domain shown. Three areas of high accumulation of solute concentration are observed, each preceding a fiber (at $y \approx 11.5 \mu \mathrm{m}$ ), and with increasing concentration magnitudes as solidification proceeds from the left wall in the direction of increasing $x$. The first fiber is associated with a maximum solute concentration of $C$ $=0.85 \%$; the second and third fibers have values of $C=0.90 \%$ and $C=0.99 \%$, respectively. Note that these values of maximum concentration are not readily discerned from Fig. 3 (and subsequent plots) since the contour lines become very crowded near the fibers. 
Figure 3(b) shows the concentration distribution for the fibers spaced at $S=1.5 D$. Compared to the previous case with $S=D$, no major changes are apparent in the concentration contours around the first fiber, as the interaction of this fiber with the advancing front is unchanged. The high-concentration (peak) areas around the second and third fibers, on the other hand, are somewhat elongated. The maximum concentration in the domain has also increased from $0.99 \%$ in Fig. 3(a) $(S=D)$ to approximately $1.26 \%$ for $S=1.5 D$. The maximum values of solute concentration around each fiber have also increased slightly for $S=1.5 \mathrm{D}$.

Increasing the fiber pitch further to $S=2 D$ (Fig. 3c) again shows little change in the concentration distribution around the first fiber, and continues the elongation process of the peak and low-concentration (valley) areas around the second and third fibers. The closed contours of value $C=0.70 \%$ around the second fiber in Fig. 3(b) have opened up and stretched in Fig. 3(c). For $S=2.5 D$ (Fig. 3d) and $S=3 D$ (Fig. 3e) the contours start looking very similar suggesting that a critical spacing has been reached around $S_{c}=2.5 D$ beyond which changes in spacing (fiber pitch) do not affect the concentration fields significantly. This is the same critical spacing reported from the thermal-field studies of Guslick et al. [17].

The progression of the solidification fronts for two of the five in-line alumina fiber configurations considered in Fig. 3 are shown in Figs. 4(a) and 4(b). The fronts are shown at equal time intervals of $0.0391 \mathrm{~s}$ to provide a common visual comparison of their locations for the different fiber spacing. Due to the lower fiber thermal conductivity relative to that of the matrix, the fronts curve toward the fibers as they approach them. This was also shown in the thermalfield studies of $[12,13,17]$. The front shape and speed is one of the factors that influences the concentration level in the solidified material. When the front moves rapidly, concentrations are higher, and vice versa. For alumina fibers the front is alternately accelerated and decelerated as 
it passes by each fiber. This results in the solute-deficient "shadows" immediately to the right of each fiber, and contributes to the solute-rich bands immediately to the left of each fiber (Fig. 3).

The concentration profiles for three inline copper fibers are shown in Fig. 5. The solidification times in the sections of the domain shown are shorter for copper fibers than for alumina fibers. They range from $0.2344 \mathrm{~s}$ to $0.5470 \mathrm{~s}$ for copper fibers and from $0.3907 \mathrm{~s}$ to $0.7033 \mathrm{~s}$ for alumina fibers. As with the observed behavior for alumina fibers (Fig. 3), there is very little change in concentration around the first fiber as the pitch is increased to $3 \mathrm{D}$ and beyond. A comparison of the solute concentrations in the presence of copper fibers against those with alumina fibers reveals two trends. Higher maximum concentrations are found in the domain with copper fibers: for example, with $S=D$ (Fig. 5a), the maximum solute concentration in the domain with copper fibers was $2.32 \%$, compared to a lower maximum of $0.99 \%$ with alumina fibers. It may also be observed that the peak concentration around each fiber increases with increasing distance into the domain for alumina fibers, but this increase is not very pronounced for copper fibers. Also, as will be shown in schematic figures to be presented, the peak concentration regions are more concentrated in angular space around the alumina fibers, but more widespread around the copper fibers.

Sample solidification fronts for the inline copper fiber case are shown in Figs. 4(c) and 4(d). Due to the higher thermal conductivity of the fibers relative to the matrix, the fronts curve away from the fibers as they approach them. This is in contrast with the case for the (lowconductivity) alumina fibers (Figs. 4a and b).

Yet another effect of the fiber properties on the solidified material is brought out by comparing Figs. 3 and 5. The solute-rich areas around copper fibers are narrower in front and cover more of the fiber circumference (over one quarter of the fiber), compared to only an eighth of the alumina fiber. In addition to fiber spacing, the fiber thermophysical properties play a 
major role in explaining these solute deposition mechanisms. The thermal conductivity and heat capacity of the fibers have an important role in determining the shape and speed of propagation of the solidification front. These, in turn, strongly influence the final solute concentration distribution in the solidified domain. In all cases, the front starts out being nearly vertical and as it approaches the first fiber (around $x=9 \mu \mathrm{m}$ ), and bends towards or away from it depending on the thermal properties of the fiber. As it contacts the fiber, it is either decelerated (alumina fibers, Fig. 4a and 4b) or accelerated (copper fibers, Fig. 4c and 4d). The reverse is true when the front leaves the fiber. For the case of alumina fibers with $S=1 D$, the velocity of the front at the top of the first fiber was estimated to be around $207 \mu \mathrm{m} / \mathrm{s}$ (compared to only $90 \mu \mathrm{m} / \mathrm{s}$ at the bottom). The acceleration of the front accounts for the dumping of excess solute at the left side of the first alumina fiber. As the local front speed $v$ increases, the diffusion layer $\delta=D_{l} /\left(\kappa_{P} v\right)$ [21] decreases, leading to greater solute buildup with less diffusion at that location.

The geometry, and the resulting confinement of the melt between the advancing front and the fiber, are also important determinants of the concentrations in the solidified material. When the top end of the solidification front approaches an alumina fiber, the front speeds up and rejects solute. Because of the relatively lower thermal conductivity of the alumina fiber, it acts as a heat insulator and the curved ends of the front are almost perpendicular to the fiber surface. As the front advances while in contact with the fiber, the solute continues to be rejected in a direction perpendicular to the front and around the fiber. Some of this solute is deposited but most of it is diffused away from the interface. The majority of solute deposition occurs when the melt gets trapped between the advancing front and the fiber. By comparison, the copper fiber has two favorable mechanisms for deposition. The curving away (convexity) of the front from the fiber allows rejection of the solute perpendicular to the front and towards the fiber as seen in Fig. 5. 
In addition, due to the high fiber thermal conductivity, the heat flow rate away from the fiber is high. This allows the layer of melt that comes into contact with the fiber to readily solidify.

To aid in visualizing the regions of high and low solute levels in the solid, Fig. 6 shows regions of solute concentrations above $0.80 \%$ (shaded) for alumina and copper fibers, at a pitch of $S=2 D$. These results have been mirrored about the symmetry planes (See Figs. $1 \mathrm{~b}$ and $1 \mathrm{c}$ ) to better display the concentration field. For alumina fibers (Fig. 6a), the high-concentration bands span much of the (horizontal) space between consecutive fibers in a row, except for the first fiber. In contrast, copper fibers (Fig. 6b) have high-concentration bands that are narrower in the regions between fibers. The high-concentration bands are also denser and steeper with copper fibers, with a maximum peak solute concentration of $2.62 \%$. This suggests that higher conductivity fibers tend to "pinch" the concentration bands near the fibers.

\section{Staggered Fibers: Effect of Longitudinal Spacing}

The simulations in this section elucidate the concentration contours at different fiber pitch (longitudinal spacing) values in a staggered fiber layout. The domain for this case is shown in Fig. 2(b) and the mesh in Fig. 2(c). Two rows of fibers are stacked on top of each other and spaced at a transverse distance $H$ between fiber centers. The top row consists of three fibers as in the inline case, and on the opposite wall, an additional row of three fibers is present with fiber centers shifted to lie between those of the upper row. The values of fiber pitch tested were $S=3$, 4 and $5 D$ corresponding to fiber volume fractions of $17.4 \%, 13.1 \%$ and $10.5 \%$ respectively, while the transverse spacing (domain height) $H$ was held fixed at $1.5 D$ for the simulations in this section. Again both alumina and copper fibers were considered.

Figure 7(a) depicts the solute distribution in the domain with six staggered alumina fibers

for a fiber spacing of $S=3 D$ after a time of $0.8591 \mathrm{~s}$. The maximum solute concentration in the 
domain was $1.12 \%$, recorded in the vicinity of the second and third fibers. The confined space between successive fibers for this configuration $(S=3 D)$ results in areas of high and low concentrations, which have a rounded shape confined within the fibers. These shapes get distorted as the horizontal fiber spacing is increased to $S=4 D$ as seen in Fig. 7(b). The lowconcentration areas (valleys) see the most elongation. As can be observed most clearly around the second fiber, the rounded valleys become more oval-shaped while the high-concentration areas (peaks) become narrower and of higher magnitude; thus, a widening of the valleys and elongation of the peaks occurs. This phenomenon is present in all fibers except the first. The process continues as transverse spacing increases, as is witnessed by Fig. 7(c) for $S=5 D$. A slight increase was observed in maximum solute concentration in the domain from $1.13 \%$ in Fig. 7(b) to $1.19 \%$ in Fig. 7(c). The maximum concentration recorded for this simulation occurred in front of the second and third fibers. The higher concentrations seen at the end of the domain near the last fiber are due to the final transient in the solidification process caused by the close proximity of the end wall resulting in an accumulation of solute. The final transient is not evident for the case shown in Fig. 7(b) since the wall is some $20 \mu \mathrm{m}$ away from the final material to solidify.

Amongst other features of the solute concentration bands in Fig. 7, one of the most prominent is a stretching phenomenon, most noticeable around the first fiber. For a fiber pitch of $3 D$, the first fiber has a band of high solute concentration starting adjacent to the left bottom quadrant of the fiber. This band extends diagonally downward to the lower boundary of the domain. Similarly there is a valley region, made up of lower concentrations around the right quadrant of the first fiber. As the fiber pitch $\mathrm{S}$ is increased to $4 D$, the single high-concentration region breaks down into two smaller regions. These regions shrink further when the fiber pitch $S$ is increased to $5 D$. Changes to the high concentration bands diminish as pitch $S$ is increased. 
The valley region, on the other hand, stretches and the original round shape becomes a sharper oval as the fiber pitch is increased. Similar changes in solute banding occur for the remaining fibers in the domain. The fiber pitch thus appears to have a major effect on shaping the concentration map, suggesting the existence of a critical fiber spacing beyond which changes in spacing have minimal effects on the concentration profile of the solidified material.

Figure 8 is a plot of the solute distribution for the corresponding copper fiber case, i.e. with transverse spacing $H$ fixed at $1.5 D$ and fiber pitch $S$ incremented. Figure 8(a) is a plot of the result for $S=3 D$ after a time $0.703 \mathrm{~s}$. The maximum concentration recorded was $3.05 \%$, and occurred around the second and third fibers. As the pitch was increased to $S=4 D$ and $5 D$, the peaks and valley regions around fibers were somewhat enlarged to occupy the additional space available. However, their shapes were preserved for the most part. These observations may be attributed to the fact that the solidification fronts with the copper fibers (see Fig. 9 and the corresponding discussion) are relatively undistorted compared to the fronts traversing alumina fibers. As a result, the deformation of the local concentration peaks and valleys around the copper fibers also exhibits much less "stretching" than for alumina fibers.

The solidification fronts for the staggered fiber layout for a representative configuration ( $S=4 D$ and $H=1.5 D$ ) are shown in Fig. 9. Figure 9(a) shows the fronts for alumina fibers. These fronts behave in a manner similar to those for inline fibers (Fig. 4) early in the solidification process. However, as solidification progresses and the front advances towards the second fiber, the front comes under the influence of two fibers simultaneously. Because the thermal conductivity of alumina is very low compared to that of the liquid melt, the fiber acts as an insulator and the temperature contours (and as a result, the fronts) are almost perpendicular to the fiber surface. This tends to distort the front, resulting in its being angled back from the vertical until it approaches the third fiber. The process is then reversed and the front is angled 
forward as it is once again under the influence of the next two fibers simultaneously. This mode of propagation continues, with the front sloping alternately backward and forward. Fig. 9(b) shows the fronts for the corresponding copper fiber case. The discussion follows along similar lines to the inline fiber case (Fig. 4), with respect to the impact of the higher conductivity fibers

on the curvature of the fronts. Here, in contrast to the alumina fiber case (Fig. 9a), the fronts never touch two fibers at the same time. This is the reason for the more localized peaks in solute concentration for the copper fiber case.

\section{Staggered Fibers: Effect of Transverse Spacing}

The final set of simulations investigated the effect of changing the transverse fiber spacing $H$ (domain height) on the solute concentration profile in the domain containing fibers arranged in a staggered layout (Fig. 2). Here, the fiber pitch was set at four times the fiber diameter $(S=4 D)$ while the transverse spacing was altered $(H=1.5,2.5$ and $3.5 D)$.

Concentrations in the solidified matrix for the alumina fibers are shown in Fig. 10. Figure 10(a) shows the concentration contours in matrix after $2.345 \mathrm{~s}$ for the case of six staggered alumina fibers with a transverse spacing of $H=1.5 D$. The maximum solute concentration in the domain was $1.13 \%$ and occurred in front of the second and third fibers. Increasing the transverse height to $2.5 D$ (Fig. 9b) begins to cause a separation of contours between vertically adjacent fibers (as can be seen for the $0.85 \%$ contour). The maximum solute concentration in the whole domain is $1.23 \%$, and again, this was centered in front of the second and third fibers. The shapes of solute-rich peaks and the low-concentration valleys are preserved for the most part and separation continues in the central part of the domain as the spacing is increased to $H=3.5 \mathrm{D}$ (Fig. 10c). The maximum solute concentration in the domain increased slightly to a value of $1.26 \%$. This value is similar to those observed for the inline fibers. As 
expected, increasing the transverse spacing of the fibers diminishes the effects of the presence of the fibers along the mid-height of the domain. Figure 11 is the corresponding case for copper fibers. The results show that the interaction between the solute bands is only significant for the lowest $H$ (Fig. 11a). As the transverse spacing is increased (Figs. 11b and c), the solute bands do not interact. This is because distortion of the interface shape around each fiber is much more localized for the copper fiber case (as shown in Figs. 4c-d and 9b).

Figure 12 shows regions of higher concentrations $(>0.80 \%)$ in the domain. The distance $a$ indicated in Figure 12(a) defines the thickness of the concentration band between two alumina fibers in a row. This distance was found to remain unchanged when increasing the transverse spacing $H$, as may be deduced from Fig. 9. This is due to the fact that the solidification fronts (not shown) do not change shape or speed in the horizontal region between the first and third fibers. The solute rejection and diffusion are unaffected in this region by a change in $H$. The arrows in Fig. 12(a) indicate the direction of movement of the boundaries of the band as the transverse fiber spacing is increased. This movement continues until eventually the band breaks at the location of the arrows and collapses into individual patches.

The corresponding case with copper fibers is shown in Fig. 12(b). The widest of these high-concentration bands are wrapped around the front left half of the second and third copper fibers. They are also characterized by much higher concentrations than for the alumina fibers, with values reaching up to a maximum of $3.09 \%$, which is the highest in the whole domain. By contrast the high-concentration bands cover only the front quadrant of the alumina fibers (Fig. 12a); the maximum levels do not exceed $1.26 \%$. This is explained as discussed previously by the different mechanisms by which the solute accumulation occurs for each fiber material. 


\section{CONCLUSIONS}

The solidification of metal matrix composites was studied through a highly efficient computational model based on the temperature-transforming method [18], appropriately modified to account for the presence of fibers. Species transport due to mass diffusion was solved to account for solute rejection and redistribution at the interface. Due to the large fiber volume fraction and very small length scales, thermal and solutal convection were not considered in this study.

Results were obtained for a variety of transverse and longitudinal fiber spacings, in order to assess the impact of fiber configuration on solute distribution in the solidified material. Two different fiber arrangements (inline and staggered) were examined for two different fiber materials (low-conductivity alumina and high-conductivity copper). The results elucidate the effect of fiber geometry and thermal properties on solute segregation. The key conclusions are as follows:

1. The solute concentrations in the solidified domain strongly depend on the shape and speed of propagation of the solidifying front.

2. Higher concentrations of solute were seen to accumulate around copper fibers than for alumina fibers. With an initial, uniform concentration of $1.0 \mathrm{wt} \% \mathrm{Cu}$ in the melt, the maximum-recorded solute concentration in the domain for alumina fibers was $1.26 \%$ while that for copper fibers was $3.11 \%$.

3. Solute-rich bands were identified between fibers of consecutive rows for both alumina and copper fibers in an inline configuration. For alumina fibers, these bands extend over almost all the space between fibers in a row. Bands with copper fibers, in contrast, do not span the distance between concentric fibers. The high-concentration bands with copper fibers also 
feature higher concentrations than with alumina, and are swept back in the direction of the advancing front.

4. In a staggered arrangement of fibers, isolated patches of high concentration $(C \geq 0.80 \%)$ in wing-like shapes are found around copper fibers, while these bands are more like flattened ovals in front of the alumina fibers (as illustrated in Fig. 12).

5. Mechanisms are suggested for the solute concentration patterns observed in the results. One mechanism is based on the confining effect when melt is trapped between the advancing front and the fiber, while the other depends on the attraction (alumina) or repulsion (copper) of the front to the fiber depending on the fiber properties relative to those of the matrix.

6. The local maximum solute concentrations around the fibers in general increased in the direction of front advancement, i.e., from the first to second to third fibers. The increase was more dramatic in the case of alumina fibers. As a result, the maximum solute concentration in the domain occurred in the vicinity of the second and third fibers.

7. For inline fibers, increasing the fiber pitch beyond a critical value $S_{c}$ did not change the overall shape of the local solute distribution around the fibers. The critical pitch for alumina fibers was found to be roughly $2.5 \mathrm{D}$ while that for copper was $2 D$. These critical spacings based on solute concentration patterns agree with the conclusions based on thermal considerations [17]. For the staggered fiber case, the solute fields are much more complex, and distinct critical spacings could not be discerned. 


\section{REFERENCES}

1. Rohatgi, P. K., Asthana, R, and Das, S., 1986, Solidification, structures, and properties of cast metal-ceramic particle composites, Int. Metals Rev., Vol. 31, pp. 115-139.

2. Chawla, K. K., 1987, Composite Materials, Springer Verlag.

3. Alexiades, V. and Solomon, A. D., 1993, Mathematical Modeling of Melting and Freezing Processes, Hemisphere, Washington D.C.

4. Viskanta, R., 1990, Mathematical modeling of transport processes during solidification of binary systems, JSME Int. Journal, Series II, Vol. 33, pp. 409-423.

5. Shyy, W., Thakur, S.S., Ouyang, H., Liu, J., and Blosch, E., 1997, Computational Techniques for Complex Transport Phenomena, Cambridge University Press.

6. Rohatgi, P. K., Narendranath, C. S., Khan, M. A., Asthana, R, and Sohal, M. S., 1994, Suspension of ceramic particles in liquid metals for synthesis of composites, HTD-Vol. 284/AMD-Vol. 182, Transport Phenomena in Solidification, ASME, pp. 197-202.

7. Mortensen, and Michaud, V., 1990, Infiltration of fibrous preforms by a binary alloy: Part 1 . Theory, Met. Trans, Vol. 21A, pp. 2059-2072.

8. Stefanescu, D.M., Moitra, A., Kacar, A.S. and Dhindaw, B.K., 1990, The influence of buoyant forces and volume fraction of particles on the particle pushing/entrapment transition during directional solidification of $\mathrm{Al} / \mathrm{SiC}$ and $\mathrm{Al} /$ Graphite composites, Met. Trans., Vol. 21A, pp. 231-239.

9. Mortensen, A. and Jin I., 1992, Solidification Processing of Metal Matrix Composites, International Materials Reviews, Vol 37, pp. 101 - 128.

10. Mortensen, A., Masur, L. J., Cornie, J A., and Flemings, M. C., 1989, Infiltration of fibrous preforms by a pure metal: Part 1. Theory, Met. Trans., Vol. 20A, pp. 2535-2547.

11. Masur, L. J., Mortensen, A., Cornie, J A., and Flemings, M. C., 1989, Infiltration of fibrous preforms by a pure metal: Part 2. Experiment, Met. Trans., Vol. 20A, pp. 2549-2557.

12. Khan, M. A., and Rohatgi, P. K., 1994, Numerical solution to a moving boundary problem in a composite medium, Num. Heat Transfer, Vol. 25, pp. 2009-2222.

13. Khan, M. A., and Rohatgi, P. K., 1995, Numerical solution to the solidification of aluminum in the presence of various fibers, J. Mater. Sci., Vol. 30, pp. 3711-3719.

14. Fan, J., Gibson, R., and Hageman, D., 1994, Microscopical study on heat transfer and mushy zone evolution in casting of metal matrix composites, HTD-Vol. 284/AMD-Vol. 182, Transport Phenomena in Solidification, ASME, pp. 191-196. 
15. Garimella, S. V., and Simpson, J. E., 1998, Interface propagation in the processing of metal matrix composites, Microscale Thermophys. Engineering, Vol. 2, pp. 173-188.

16. Simpson, J. E., Garimella, S. V., and Guslick, M. M., 1998, Interface propagation in the presence of a fibrous phase in alloy solidification, Heat Transfer 1998, Vol. 7, pp. 235-240.

17. Guslick, M. M., Simpson, J. E., and Garimella, S. V., 1999, Fiber-spacing effects in the solidification processing of metal matrix composites, Num. Heat Transfer, Vol. 35, pp. 587607.

18. Zeng, X. and Faghri, A., 1994, Temperature-transforming model for binary solid-liquid phase change problems Part I: Mathematical modeling and numerical methodology, Num. Heat Transfer, Vol. 25B, pp. 467-480.

19. Swaminathan, C. R. and Voller, V. R., 1997, Towards a general numerical scheme for solidification systems, Int. J. Heat Mass Transfer, Vol. 40, pp. 2859-2868.

20. Voller, V. R., Brent A. D., and Prakash, C., 1989, The modelling of heat, mass and solute transport in solidification systems, Int. J. Heat Mass Transfer, Vol. 32, pp. 1719-1731.

21. Kurz, W. and Fisher, D. J., 1989, Fundamentals of Solidification, Trans Tech Publications. 


\section{FIGURE CAPTIONS}

Fig. 1 Schematics of (a) the synthesis of an MMC by infiltration, (b) the problem domain considered for in-line fibers, and (c) the computational domain with mesh.

Fig. 2 Schematics of (a) the problem domain being considered for staggered fibers and (b) the computational domain with mesh.

Fig. 3 Solute concentration profiles for aluminum alloy solidification with three in-line alumina fibers: (a) $S=1 \mathrm{D}$ at $0.3907 \mathrm{~s}$, (b) $\mathrm{S}=1.5 \mathrm{D}$ at $0.3907 \mathrm{~s}$, (c) $\mathrm{S}=2 \mathrm{D}$ at $0.5176 \mathrm{~s}$, (d) $\mathrm{S}=2.5 \mathrm{D}$ at $0.5860 \mathrm{~s}$, and (e) $\mathrm{S}=3 \mathrm{D}$ at $0.7033 \mathrm{~s}$.

Fig. 4 Front locations for alloy solidification with three in-line fibers. The time interval between fronts is $0.0391 \mathrm{~s}$. Alumina fibers: (a) $S=1.5 D$, (b) $S=3 D$; copper fibers: (c) $S$ $=1.5 D,(\mathrm{~d}) S=3 D$.

Fig. 5 Solute concentration profiles for alloy solidification with three in-line copper fibers: (a) $\mathrm{S}=1 \mathrm{D}$ at $0.2344 \mathrm{~s}$, (b) $\mathrm{S}=1.5 \mathrm{D}$ at $0.2735 \mathrm{~s}$, (c) $\mathrm{S}=2 \mathrm{D}$ at $0.3126 \mathrm{~s}$, (d) $\mathrm{S}=2.5 \mathrm{D}$ at $0.4298 \mathrm{~s}$, and (e) $\mathrm{S}=3 \mathrm{D}$ at $0.5470 \mathrm{~s}$.

Fig. 6 Domain showing solute-rich bands. Bands are shown at concentrations higher than $0.80 \%$ for (a) alumina and (b) copper fibers $(S=2 D, H=1.5 D)$.

Fig. 7 Solute concentration profiles for alloy solidification with six staggered alumina fibers: (a) $S=3 D$ at $0.8591 \mathrm{~s}$, (b) $S=4 D$ at $2.3445 \mathrm{~s}$, and (c) $S=5 D$ at $2.3445 \mathrm{~s}$. The transverse fiber spacing is fixed at $H=1.5 \mathrm{D}$.

Fig. 8 Solute concentration profiles for alloy solidification with six staggered copper fibers: (a) $S=3 D$ at $0.7033 \mathrm{~s}$, (b) $S=4 D$ at $2.1868 \mathrm{~s}$, and (c) $S=5 D$ at $2.3445 \mathrm{~s}$. The transverse fiber spacing is fixed at $H=1.5 D$.

Fig. 9 Sample front locations for (a) alumina and (b) copper fibers for $S=4 D$. $H$ is fixed at $1.5 D$

Fig. 10 Solute concentration profiles for alloy solidification with six-staggered alumina fibers at time $2.3446 \mathrm{~s}$. The fiber pitch is fixed at $S=4 D$ and the transverse fiber spacings $(H)$ used are (a) 1.5, (b) 2.5, and (c) 3.5 fiber diameters.

Fig. 11 Solute concentration profiles for alloy solidification with six-staggered copper fibers at time $2.1868 \mathrm{~s}$. The fiber pitch is fixed at $S=4 D$ and the transverse fiber spacings $(H)$ used are (a) 1.5, (b) 2.5, and (c) 3.5 fiber diameters.

Fig. 12 Regions with bands of high concentrations with six staggered (a) aluminum and (b) copper fibers. $S=4 D, H=3.5 D$. Shaded areas have concentrations $C>0.8 \%$. 


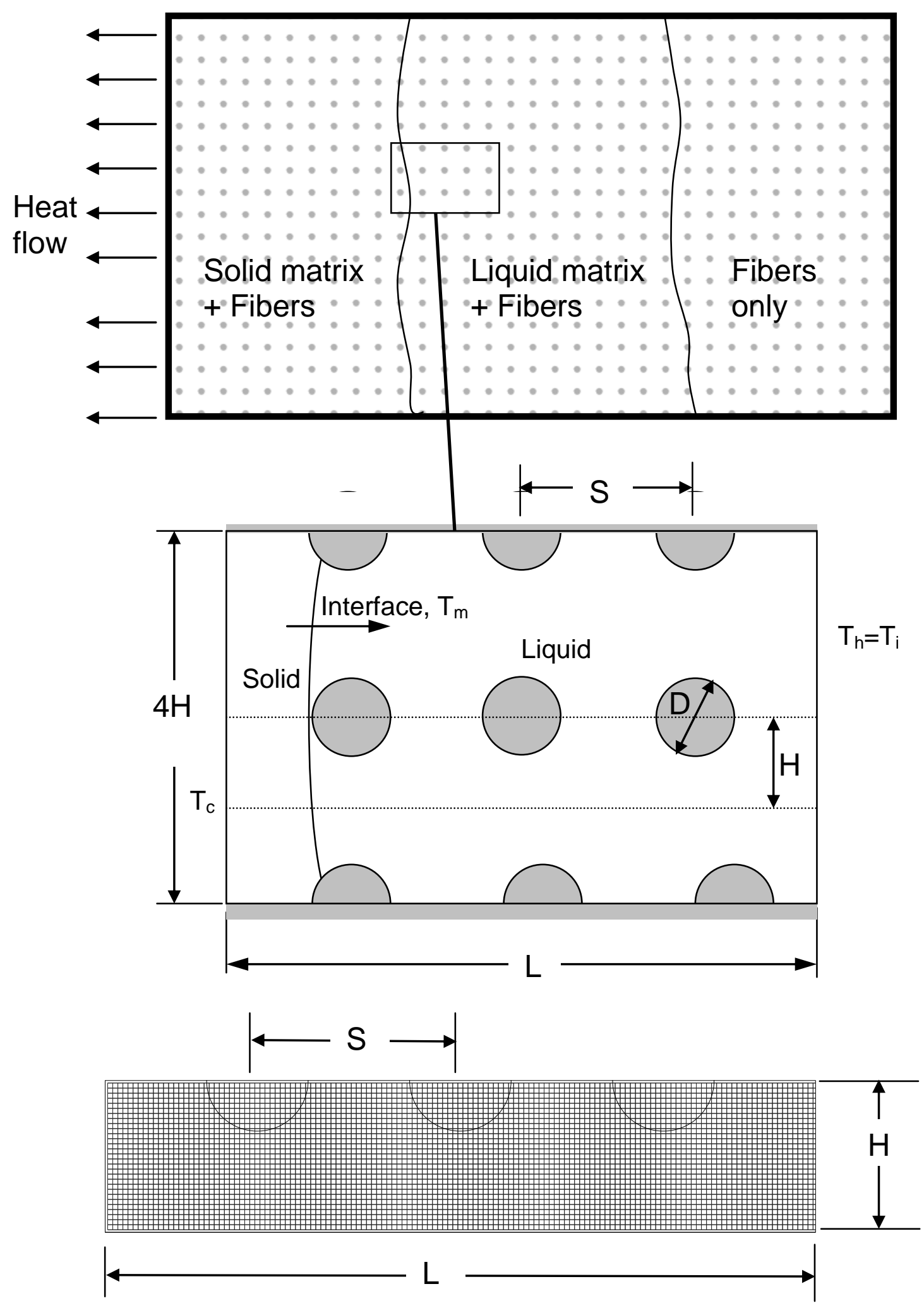

(a)

(b)

(c)

Fig. 1, Moussa et al. 


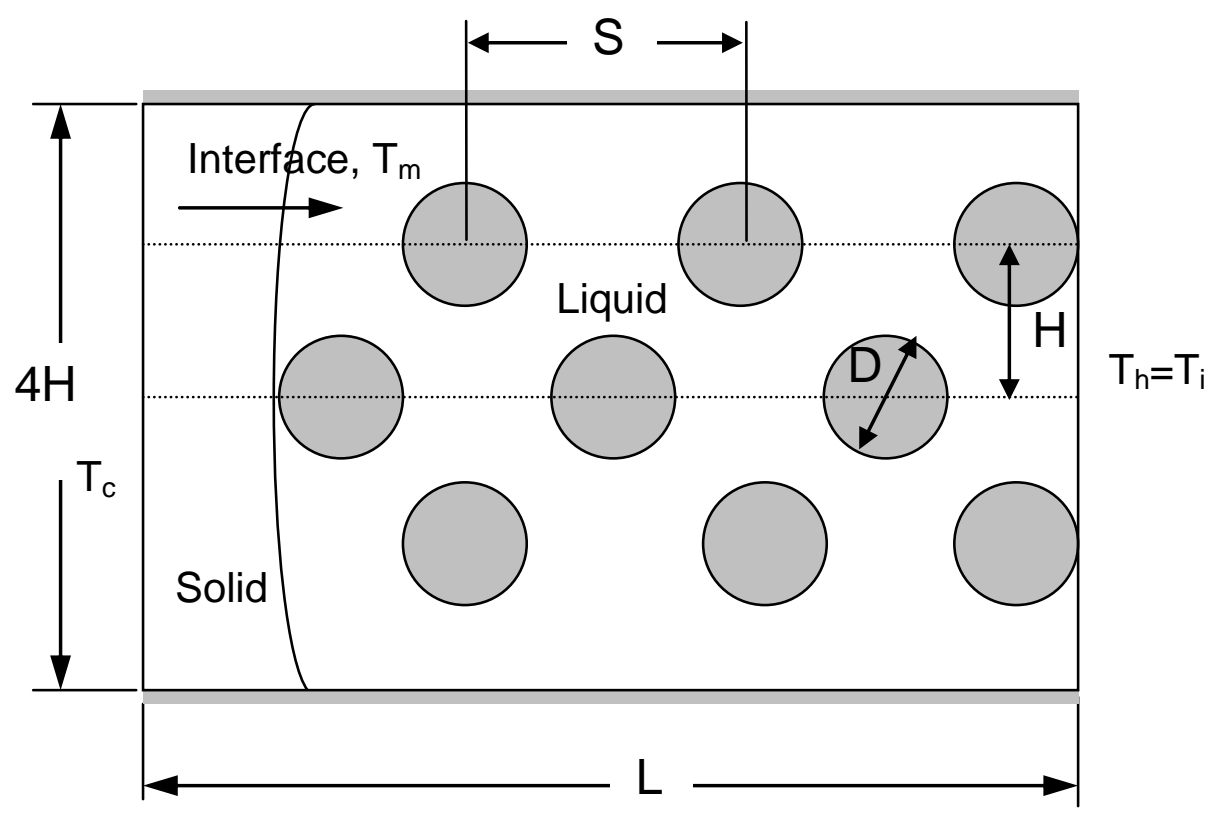

(a)

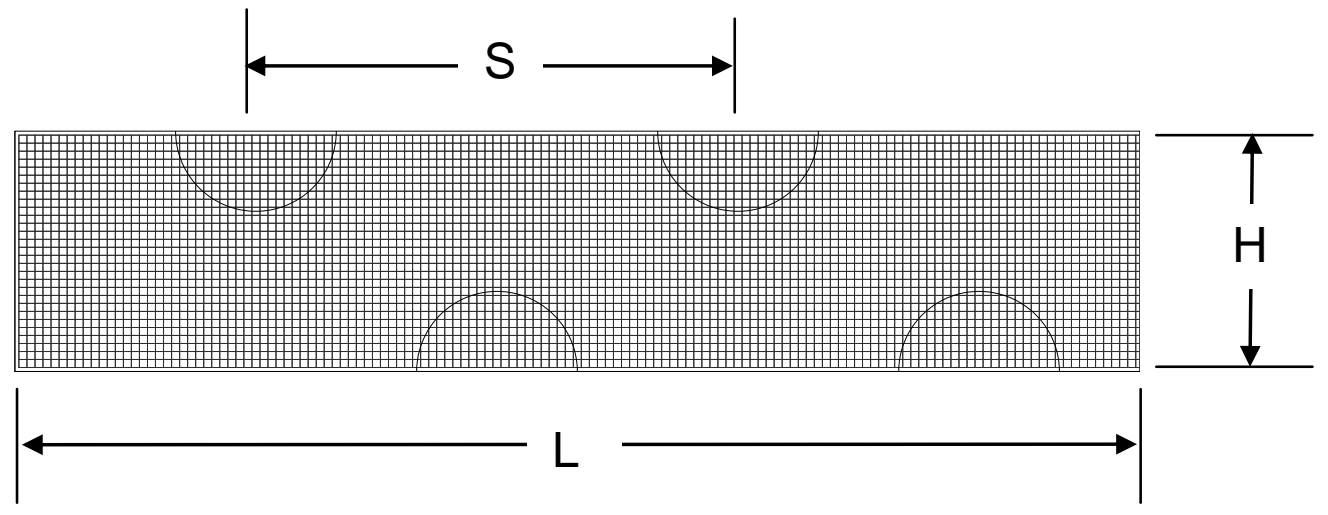

(b)

Fig. 2, Moussa et al. 


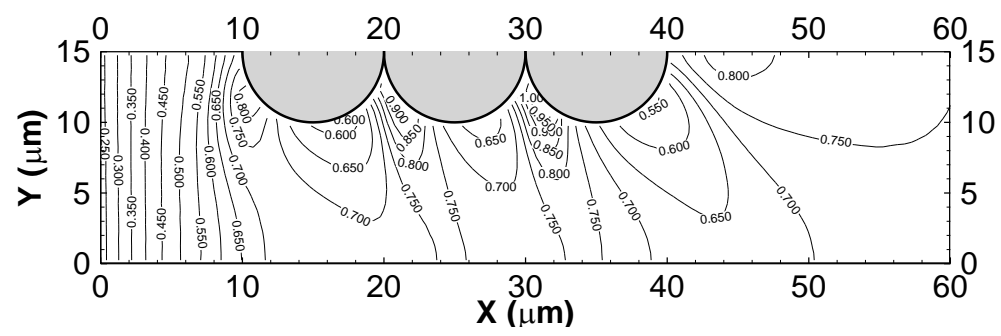

(a)

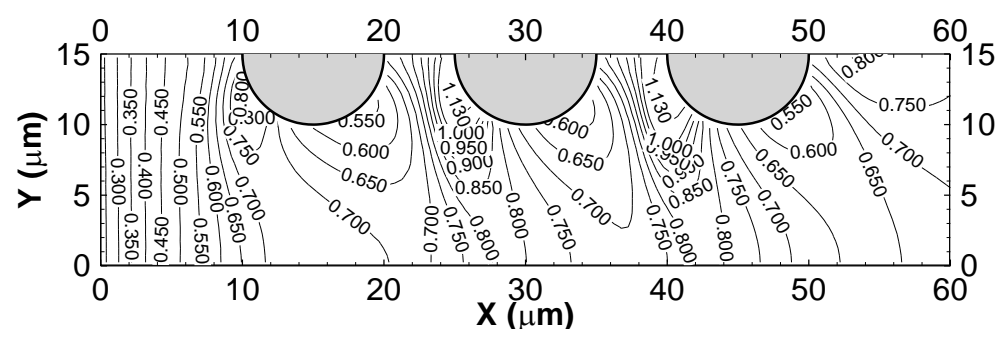

(b)

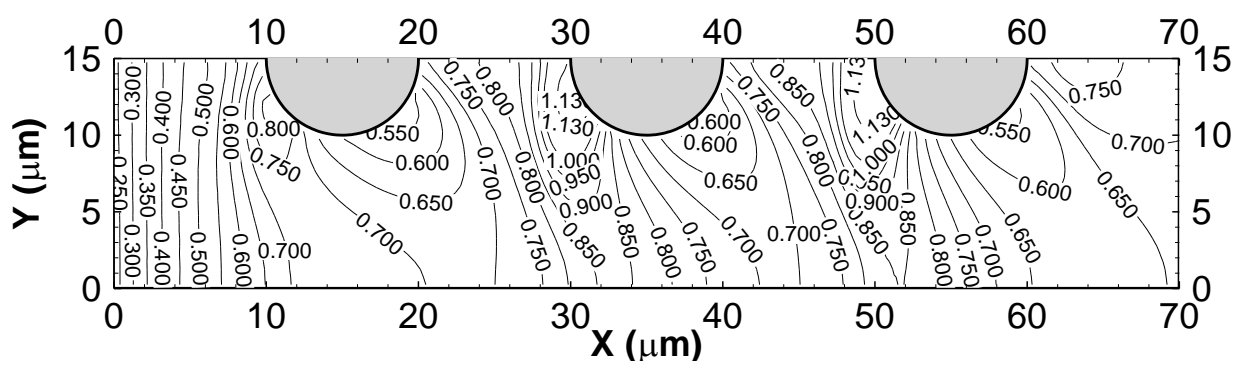

(c)

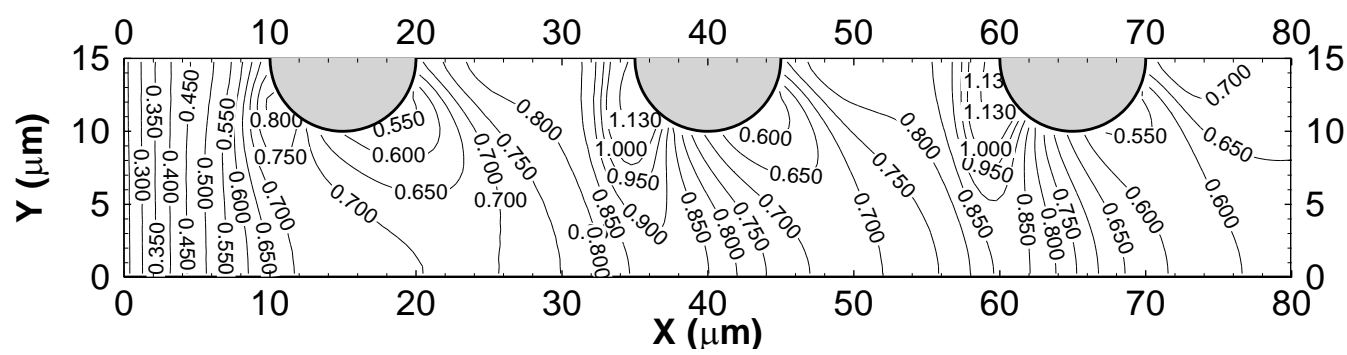

(d)

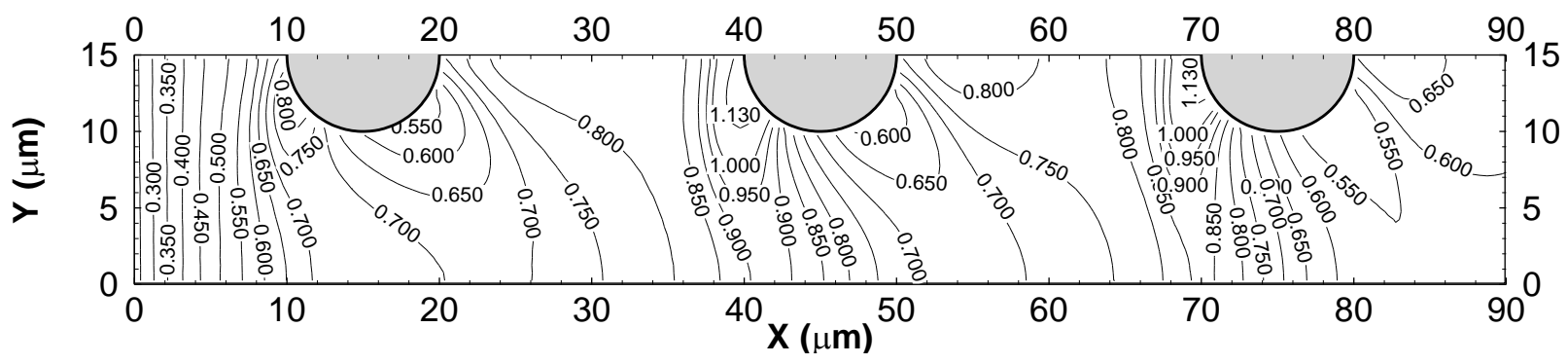

(e)

Fig. 3, Moussa et al. 


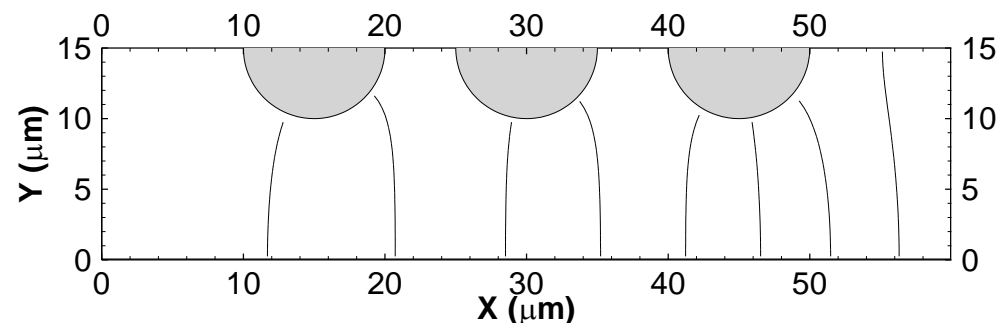

(a)
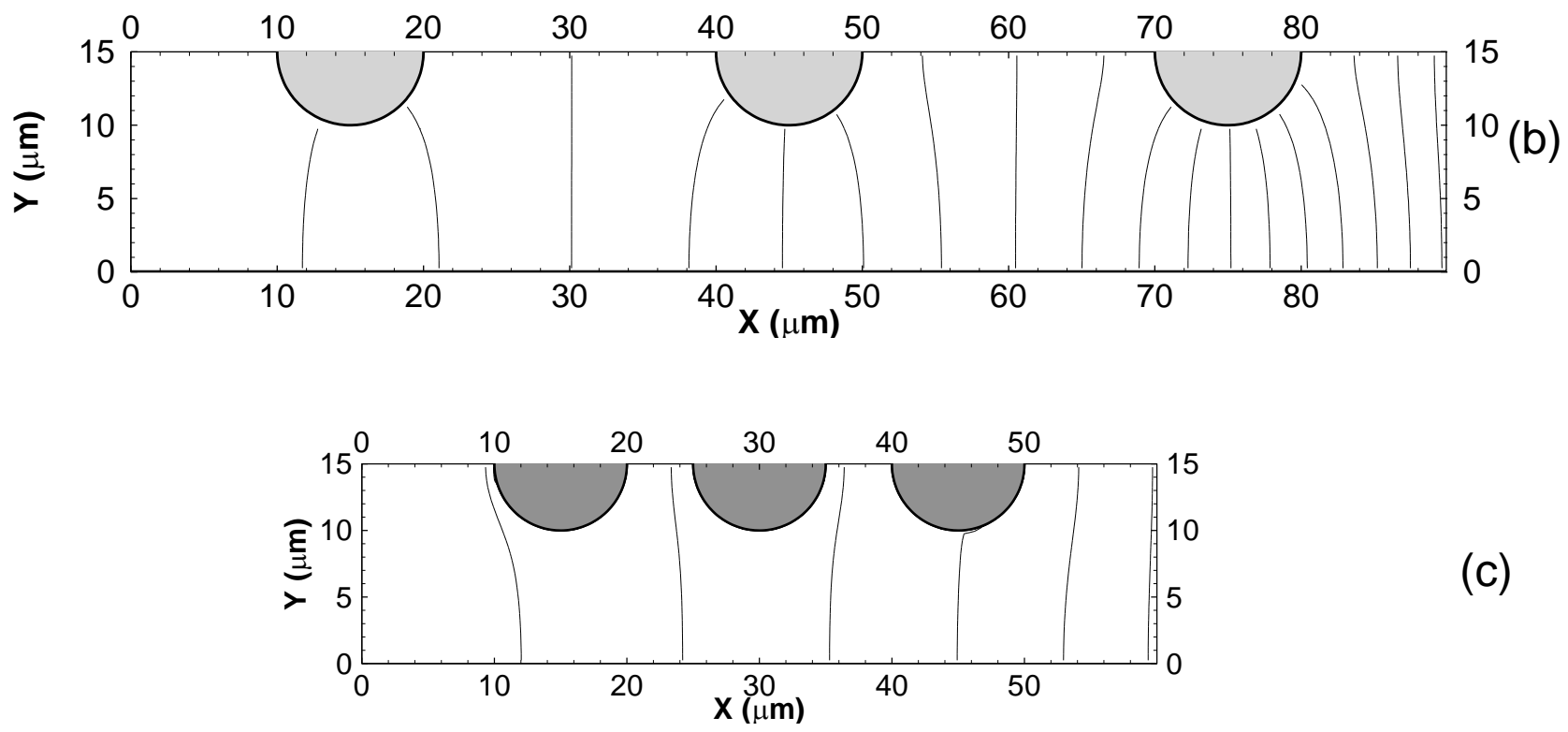

(c)

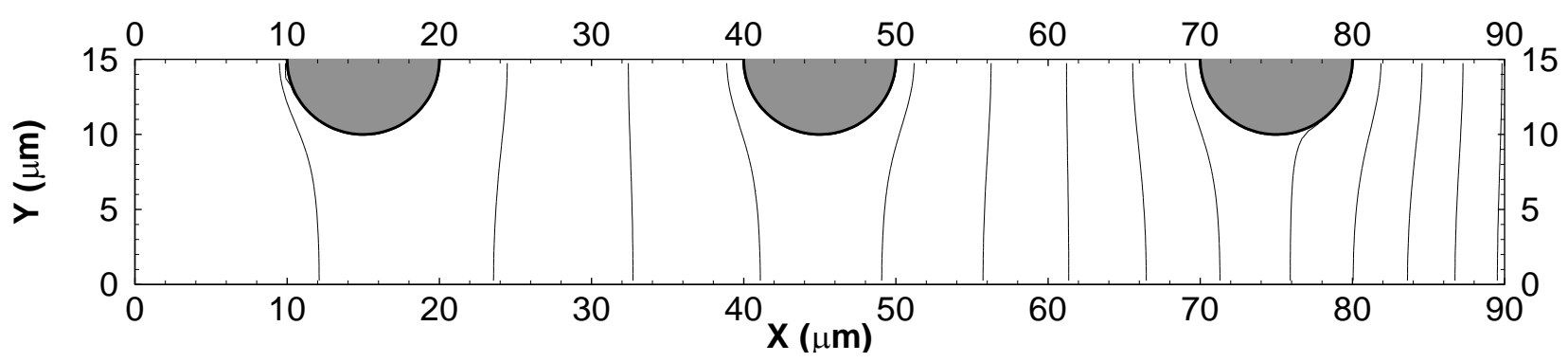

(d)

Fig. 4, Moussa et al. 


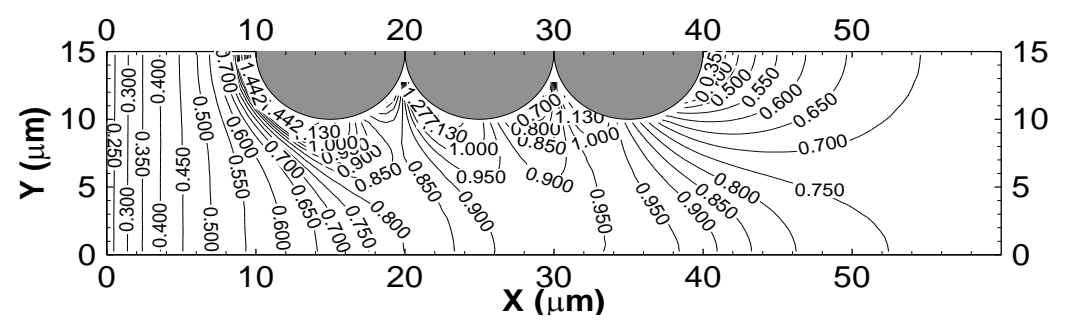

(a)

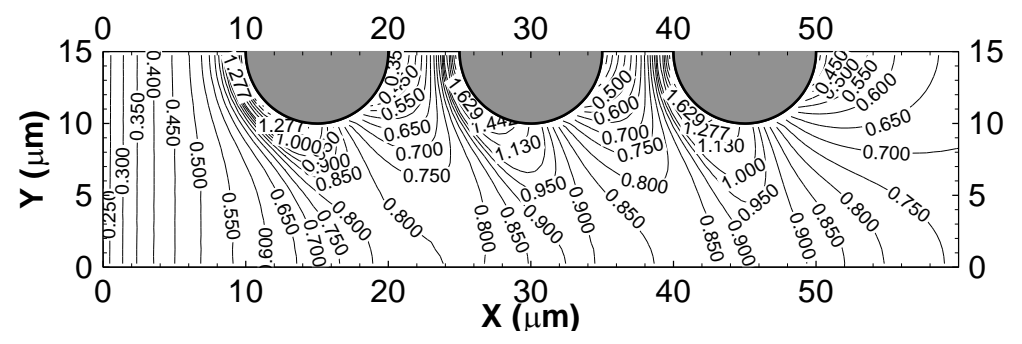

(b)

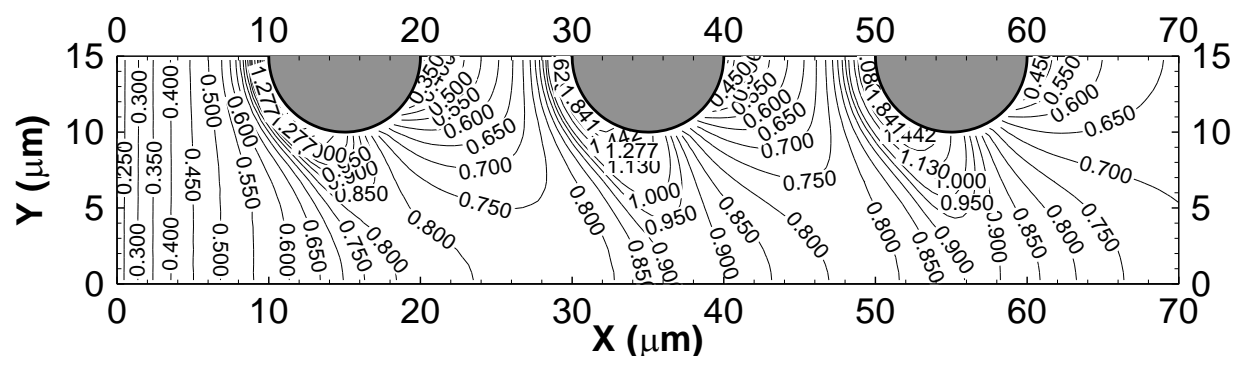

(c)

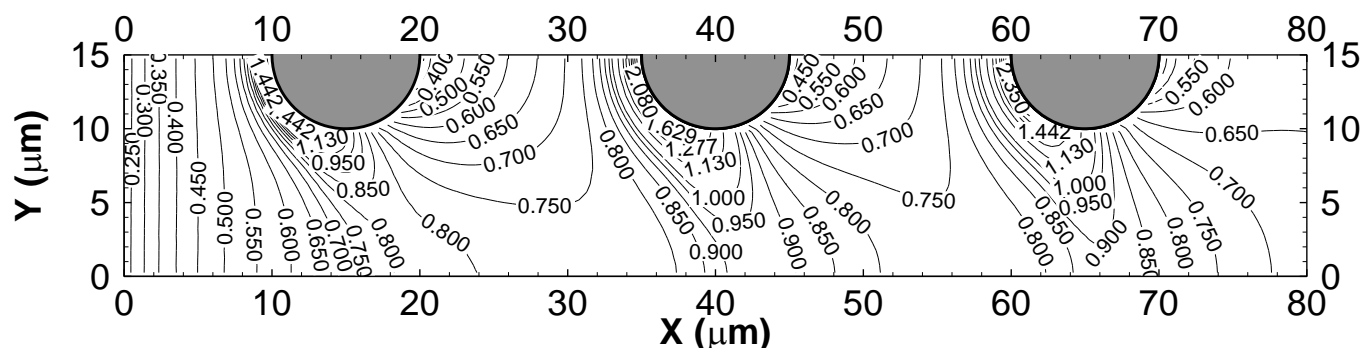

(d)

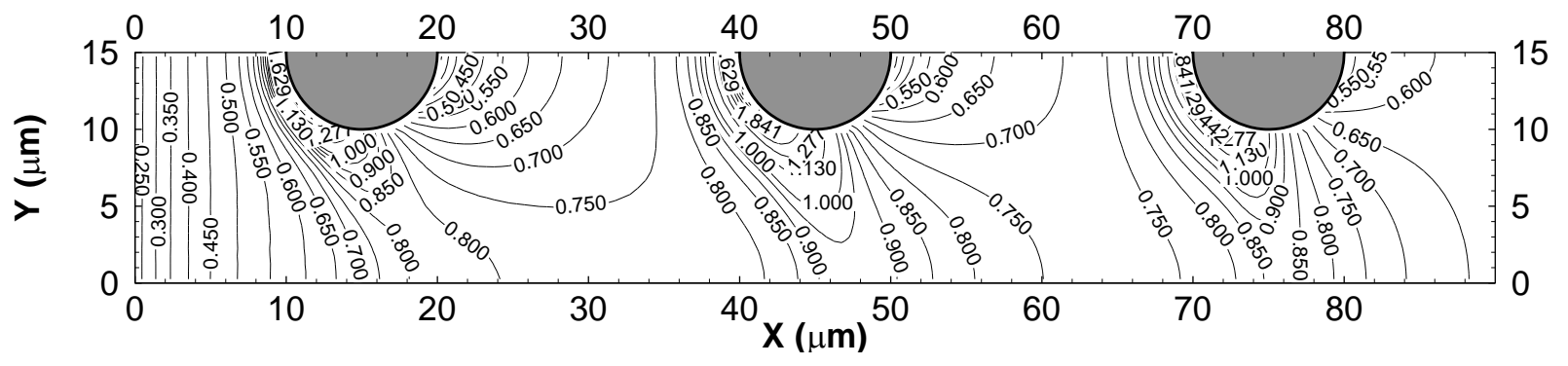

Fig. 5, Moussa et al. 


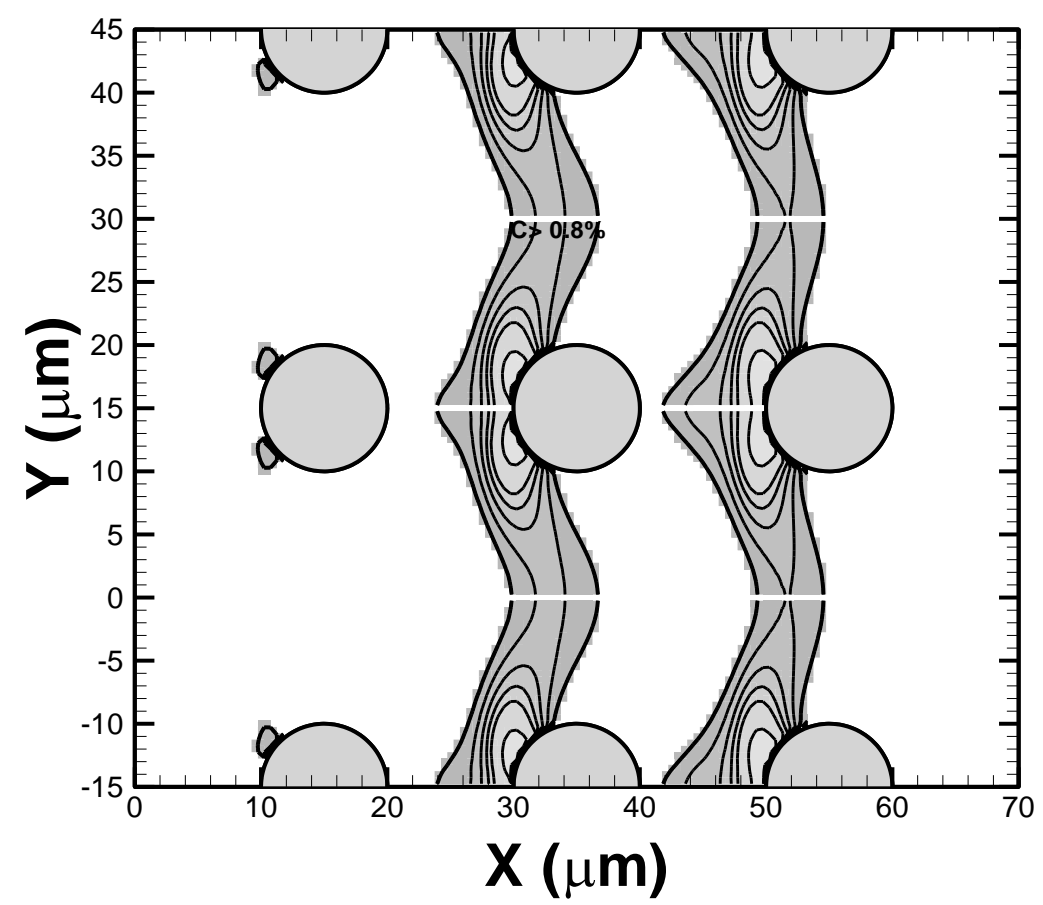

(a)

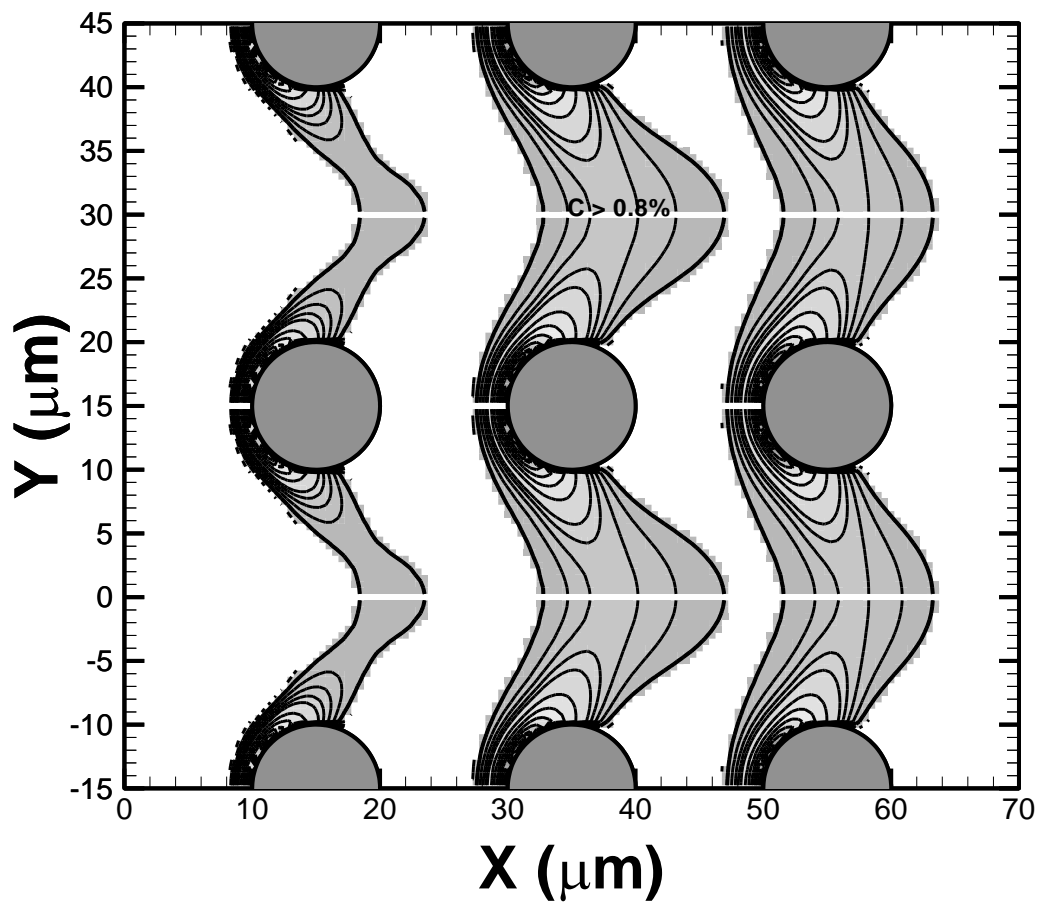

(b)

Fig. 6, Moussa et al. 


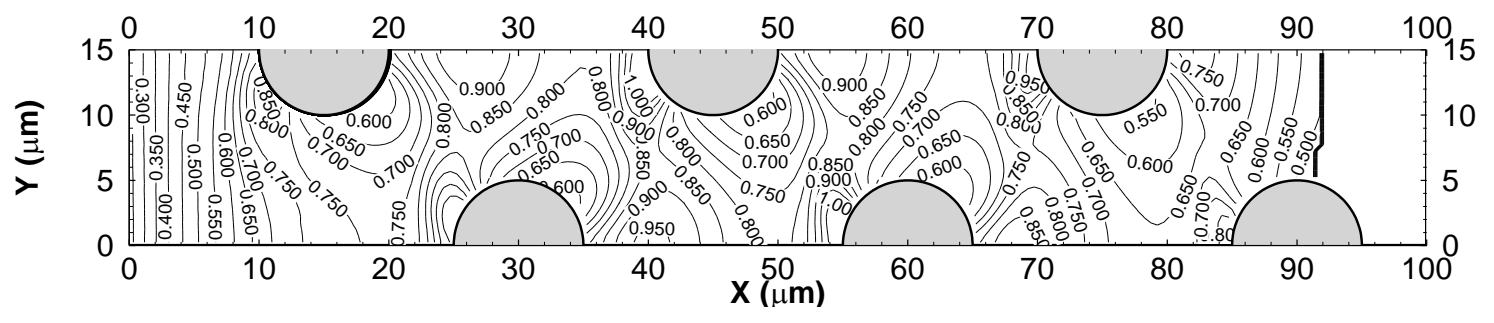

(a)
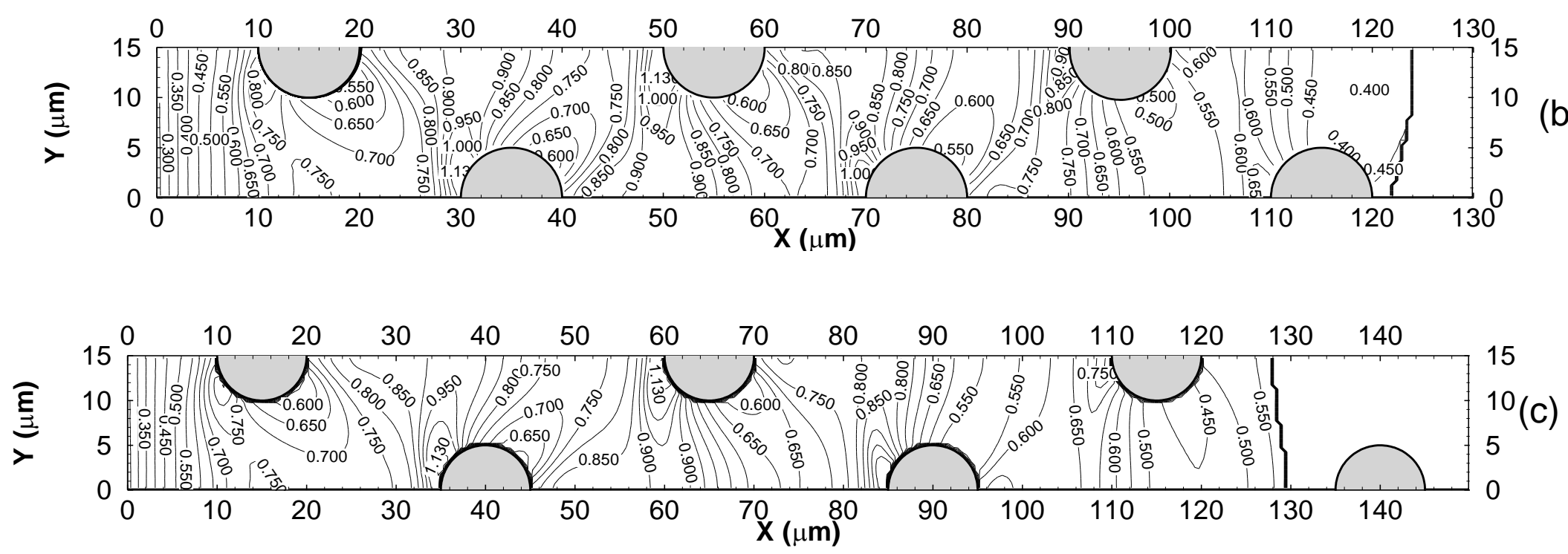

Fig. 7, Moussa et al. 

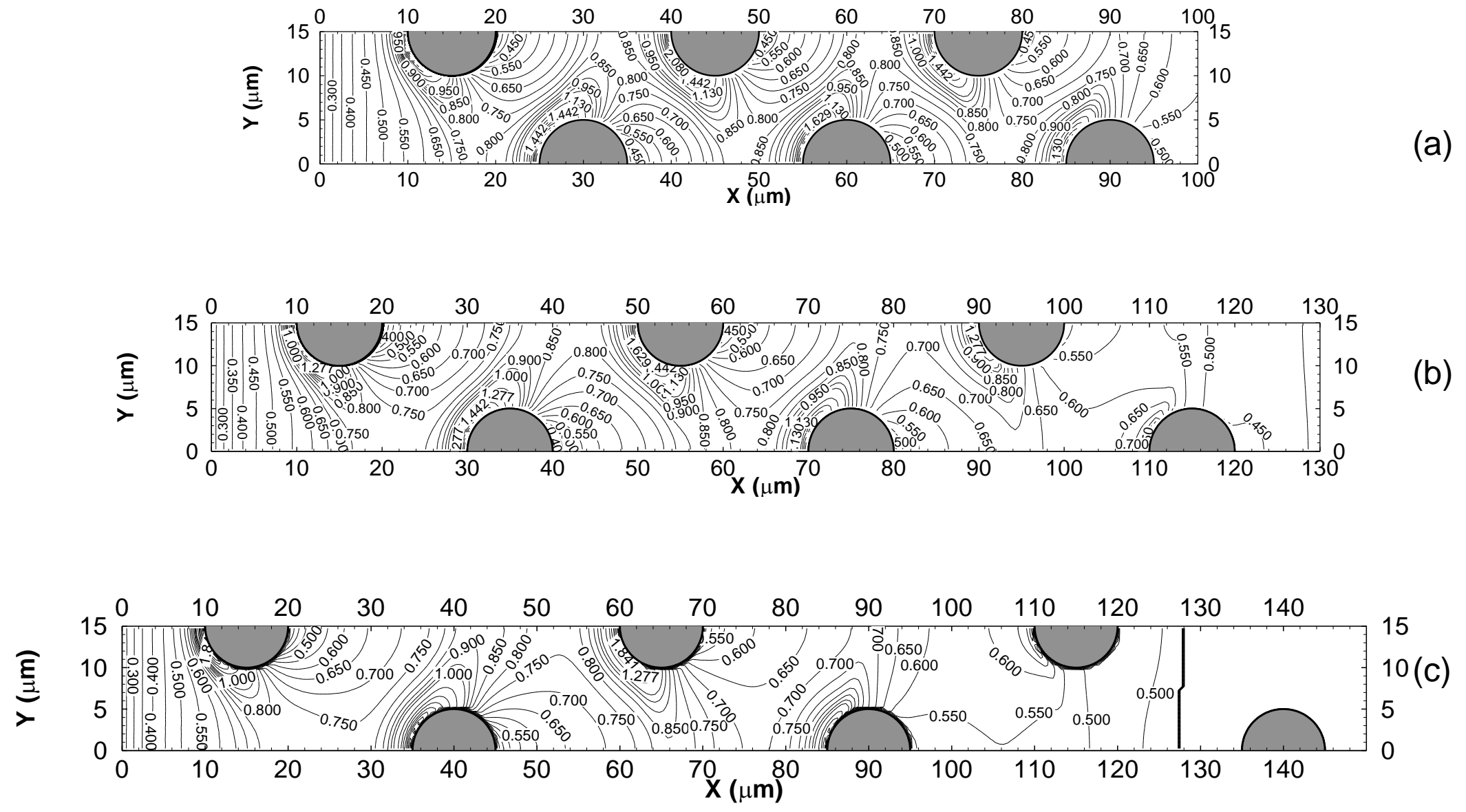

Fig. 8, Moussa et al. 


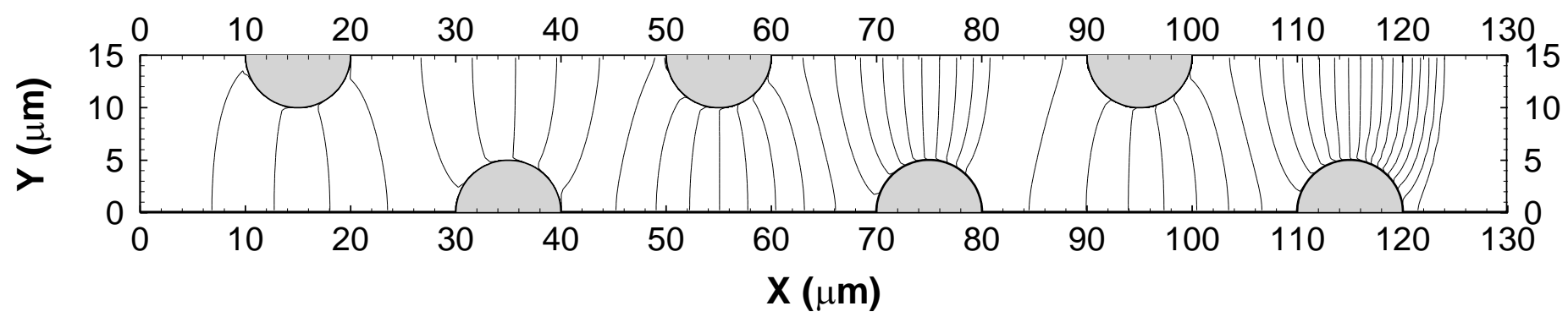

(a)

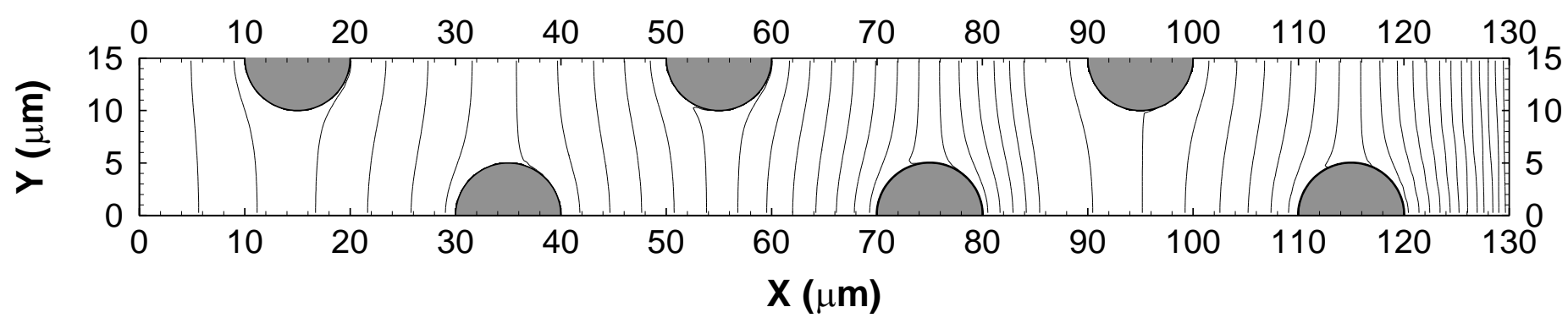

(b)

Fig. 9, Moussa et al. 


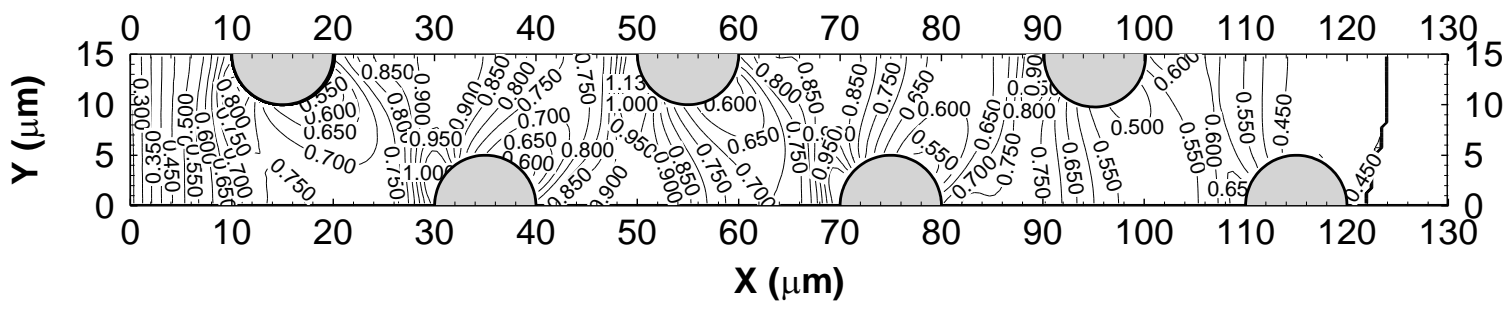

(a)
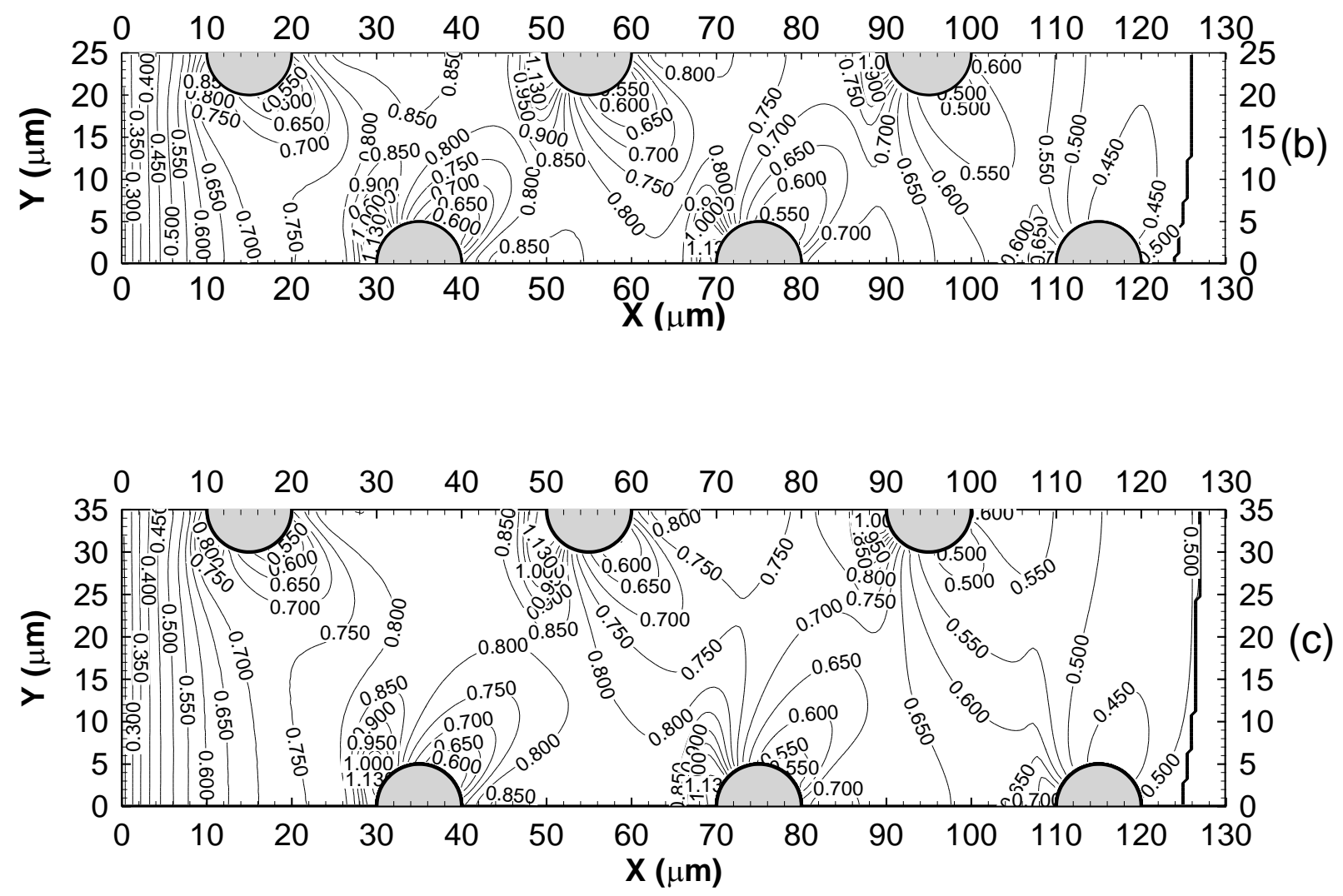

Fig. 10, Moussa et al. 


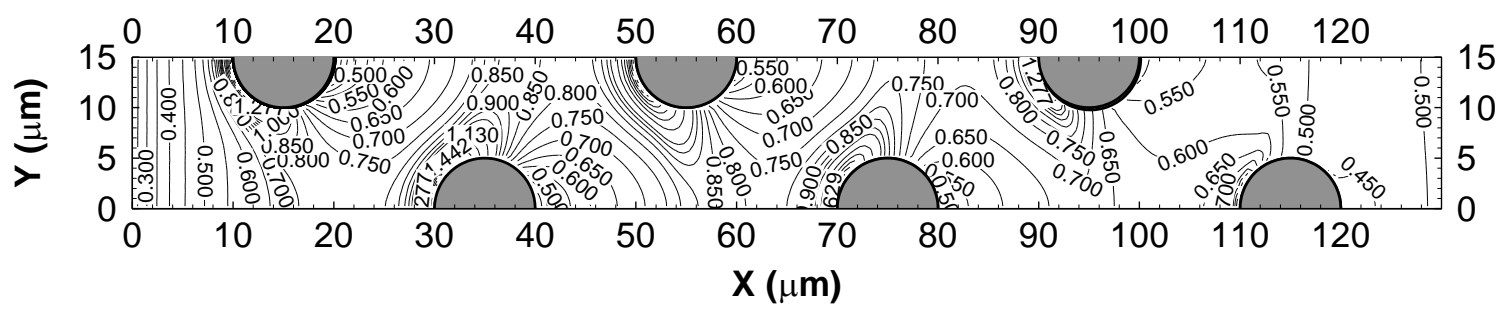

(a)

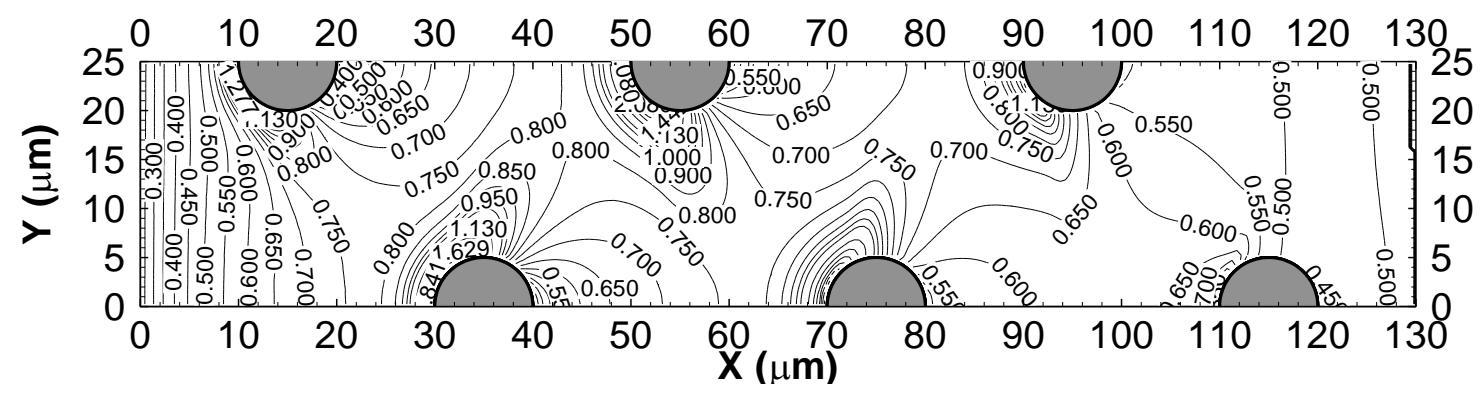

(b)

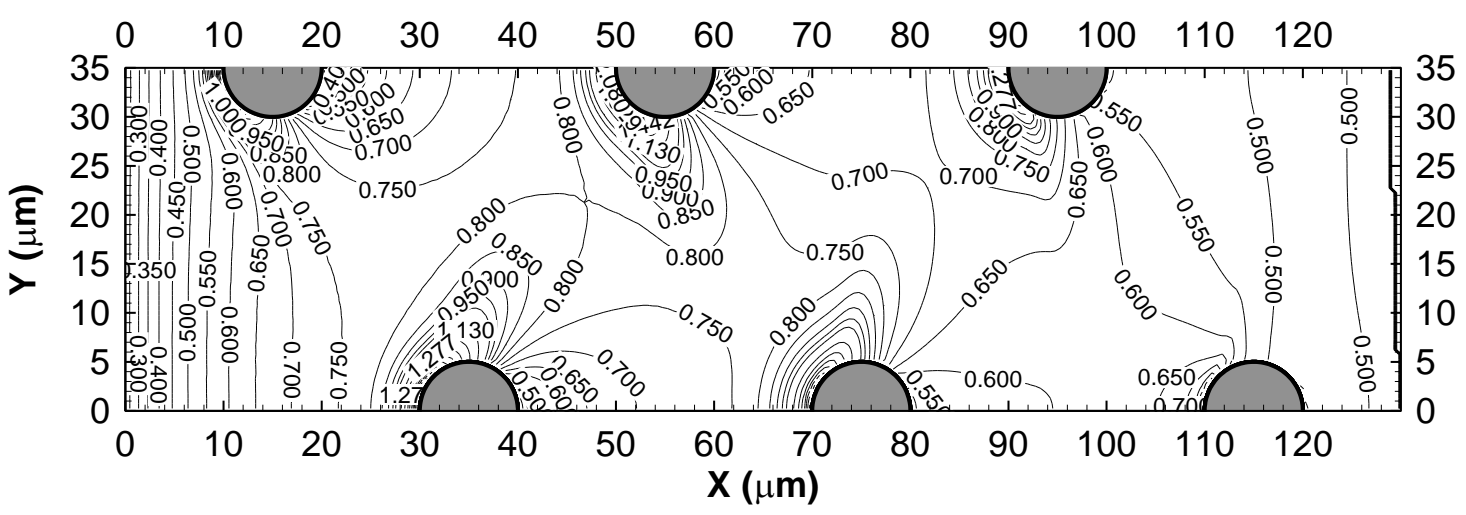

(c)

Fig. 11, Moussa et al. 


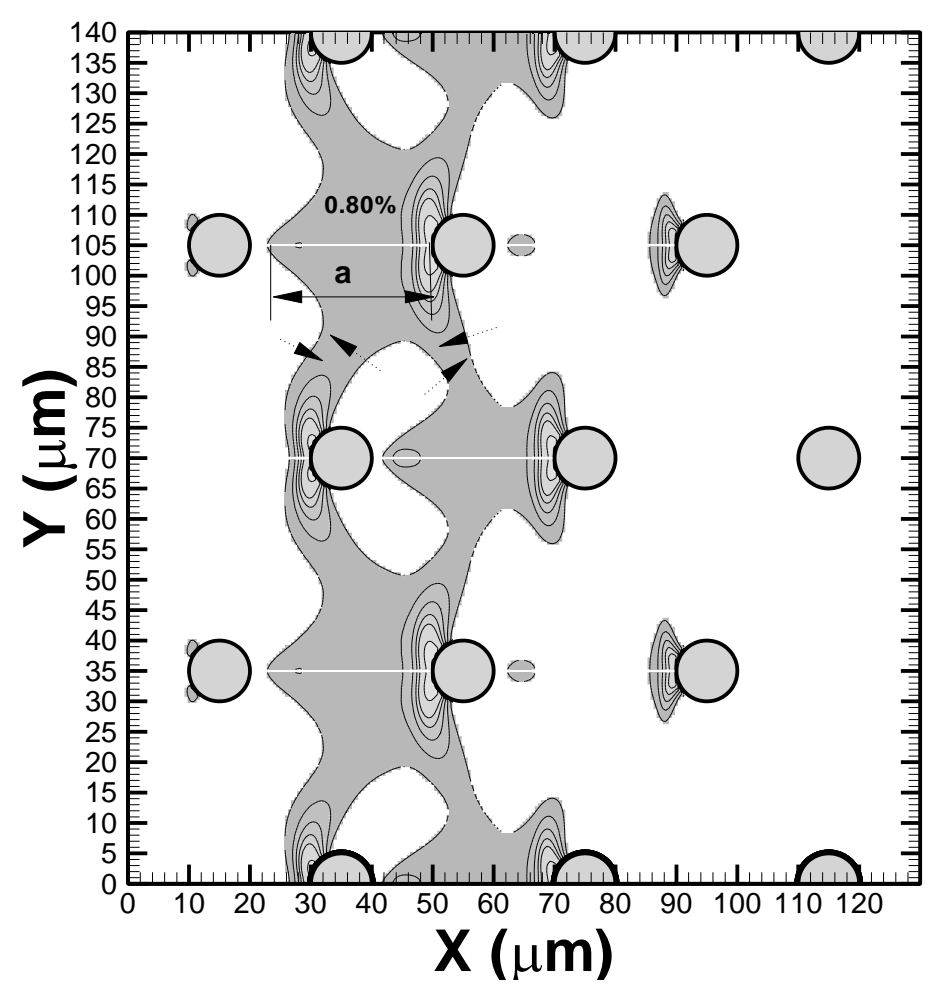

(a)

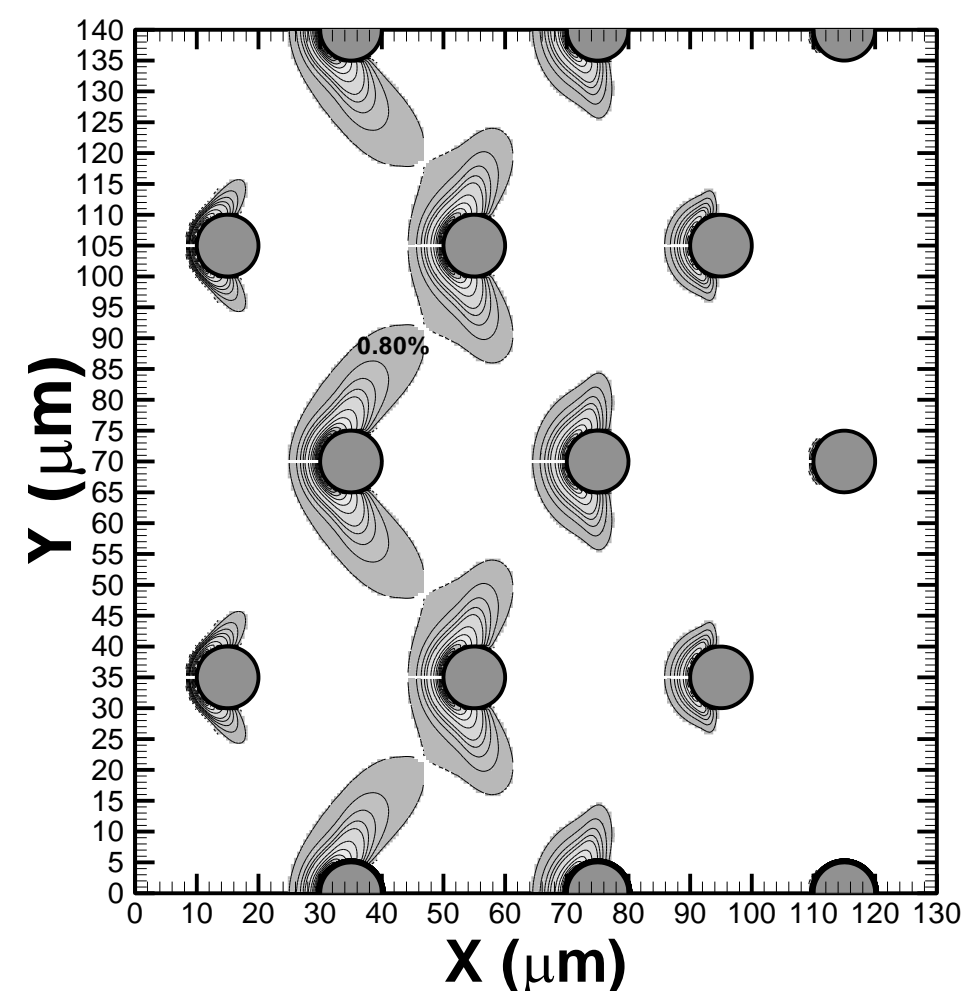

(b)

Fig. 12, Moussa et al. 